\title{
Echinacea purpurea (L.) Moench treatment of monocytes promotes tonic interferon signaling, increased innate immunity gene expression and DNA repeat hypermethylated silencing of endogenous retroviral sequences
}

Ken Declerck', Claudina Perez Novo ${ }^{1}$, Lisa Grielens ${ }^{1}$, Guy Van Camp², Andreas Suter ${ }^{3}$ and Wim Vanden Berghe ${ }^{1 *}$ (D)

\begin{abstract}
Background: Herbal remedies of Echinacea purpurea tinctures are widely used today to reduce common cold respiratory tract infections.

Methods: Transcriptome, epigenome and kinome profiling allowed a systems biology level characterisation of genomewide immunomodulatory effects of a standardized Echinacea purpurea (L.) Moench extract in THP1 monocytes.

Results: Gene expression and DNA methylation analysis revealed that Echinaforce ${ }^{\oplus}$ treatment triggers antiviral innate immunity pathways, involving tonic IFN signaling, activation of pattern recognition receptors, chemotaxis and immunometabolism. Furthermore, phosphopeptide based kinome activity profiling and pharmacological inhibitor experiments with filgotinib confirm a key role for Janus Kinase (JAK)-1 dependent gene expression changes in innate immune signaling. Finally, Echinaforce ${ }^{\circledR}$ treatment induces DNA hypermethylation at intergenic $\mathrm{CpG}$, long/short interspersed nuclear DNA repeat elements (LINE, SINE) or long termininal DNA repeats (LTR). This changes transcription of flanking endogenous retroviral sequences (HERVs), involved in an evolutionary conserved (epi) genomic protective response against viral infections.
\end{abstract}

Conclusions: Altogether, our results suggest that Echinaforce ${ }^{\circledast}$ phytochemicals strengthen antiviral innate immunity through tonic IFN regulation of pattern recognition and chemokine gene expression and DNA repeat hypermethylated silencing of HERVs in monocytes. These results suggest that immunomodulation by Echinaforce ${ }^{\oplus}$ treatment holds promise to reduce symptoms and duration of infection episodes of common cold corona viruses (CoV), Severe Acute Respiratory Syndrome (SARS)-CoV, and new occurring strains such as SARS-CoV-2, with strongly impaired interferon (IFN) response and weak innate antiviral defense.

\footnotetext{
* Correspondence: Wim.vandenberghe@uantwerpen.be

'Laboratory of Protein Chemistry, Proteomics and Epigenetic Signaling (PPES), Department of Biomedical Sciences, University of Antwerp (UA), Antwerp, Belgium

Full list of author information is available at the end of the article
}

(c) The Author(s). 2021 Open Access This article is licensed under a Creative Commons Attribution 4.0 International License, which permits use, sharing, adaptation, distribution and reproduction in any medium or format, as long as you give appropriate credit to the original author(s) and the source, provide a link to the Creative Commons licence, and indicate if changes were made. The images or other third party material in this article are included in the article's Creative Commons licence, unless indicated otherwise in a credit line to the material. If material is not included in the article's Creative Commons licence and your intended use is not permitted by statutory regulation or exceeds the permitted use, you will need to obtain permission directly from the copyright holder. To view a copy of this licence, visit http://creativecommons.org/licenses/by/4.0/ The Creative Commons Public Domain Dedication waiver (http://creativecommons.org/publicdomain/zero/1.0/) applies to the data made available in this article, unless otherwise stated in a credit line to the data. 


\section{Background}

Distinct species of the plant genus Echinacea have traditionally been used in North America against infectious diseases and wounds $[1,2]$. Currently, a wide variety of Echinacea preparations are used world-wide as complementary herbal remedy to improve the immune response to protect against common cold symptoms and influenza infections. Of all Echinacea species, Echinacea purpurea (purple coneflower) is the most popular variety used in Western countries. Different Echinacea purpurea extracts (different species, plant parts, manufacturing) or derived compounds showed antioxidant, antibacterial, antifungal, antiviral and mosquitocidal activities in cell culture experiments [3], although absolute comparisons between studies with different preparations remain difficult $[4,5]$. Complex immunomodulatory actions of Echinacea have been described including both pro- and anti-inflammatory effects $[2,3,6]$. The compounds that contribute to these activities are alkylamides, glycoproteins, polysaccharides and caffeic acid derivates that may act independently or in synergy $[1,3$, 7-9].

In this study, we evaluated Echinaforce ${ }^{\circ}$, a commercially registered herbal medicinal tincture of Echinacea purpurea (L.) Moench (A.Vogel Bioforce, Switzerland) in several European countries including Switzerland, Austria, UK, Spain, Netherland, Denmark, Finland, Sweden, Slovenia, as well as Canada. The tincture contains 5\% root extract and $95 \%$ herb extract following extraction with $65 \%$ ethanol V/V. Echinaforce ${ }^{\circ}$ phytochemicals reveal immune modulatory, antiinflammatory, anti-bacterial, anti-viral and antiparasitic activity [9-21]. Clinical efficacy could be shown with different batches in acute treatment [22] or for prevention [23] of respiratory tract infections. A 4month randomized, double blind, placebo-controlled study ( $n=755$ subjects, of which 376 received placebo) on the safety and efficacy of Echinaforce ${ }^{\bullet}$ to prevent common cold symptoms, showed significantly less cold episodes and of shorter duration as well as lower infection recurrence rate in the Echinaforce ${ }^{\circ}$ treated versus placebo treated group [23]. Moreover, no differences between placebo and Echinaforce ${ }^{\circ}$ group were reported in relation to health risk and safety [23]. Despite the promising immune potentiating properties of Echinaforce $^{\circ}$, the responsible molecular targets have only partially been identified, such as the cannabinoid receptor 2 (CB2) [7, 19, 24], the cAMP, p38/MAPK and JNK signaling pathways, as well as NF- $\mathrm{KB}$ and ATF2/CREB1 transcription factors. To further clarify its mode of action, we applied a system biology approach by integrating genomewide transcriptome, epigenome and kinome signaling profiles of THP1 monocytes treated with Echinaforce ${ }^{\circ}$.

\section{Methods}

\section{Cell lines and treatments}

Echinaforce ${ }^{\circ}$ (batch nr. 040070, A. Vogel Bioforce AG, Roggwil, Switzerland) is a standardized preparation obtained by ethanol extraction of freshly harvested Echinacea purpurea herb and roots (95:5). The extract Echinaforce ${ }^{\odot}$ itself is strictly produced under GMP conditions and tested therefore on different levels (seed, plant, extract, tablet, etc.) thoroughly in the same manner as an allopathic remedy since it is a registered product in Europe. The plant has been identified taxiconomically and also with a DNA test. The same seeds of this plant have been used for more than 50 years (since 1955) in the company A. Vogel to produce the standardized test item Echinaforce ${ }^{\circ}$. This means that end of year a part of the Echinacea plant is used to take the seeds in late autumn to use them for plantation next spring then. Since the majority of the Echinacea cultivation is in the vicinity of A. Vogel AG in Roggwil (Switzerland) no adulteration with other plants takes place. According to Good Agricultural Practice, for every batch used for for production of the standardized extract, the plant species is visually verified by an expert before it is released for production of the registered medicinal herbal extracts. The main basis for releases of any batch of Echinaforce is the HPLC fingerprint, TLCs and a minimum amount of the alklymide tetraen as a marker substance. The composition of marker compounds like alkylamides (i.e. those compounds known to characterize this species of Echinacea) was described previously [3, 6, 25]. With this strategy, A. Vogel can guarantee that every batch is similar in its constituents and its activity profile. Extended research on pharmacological activity with different batches have been carried out by the company showing consistent activity in in vitro settings (antiviral, immunemodulatory activity). In contrast to pressed juice extracts, Echinaforce $e^{\ominus}$ extract does not contain polysaccharides which are known to stimulate the immune system nonspecifically [26-29]. The alcohol concentration of Echinaforce ${ }^{\circ}$ tincture extract was $65 \% \mathrm{v} / \mathrm{v}$ and solvent controls have been included in all experimental in vitro experiments to rule out nonspecific effects. In addition, the preparation was free of detectable endotoxin as determined by means of a commercial assay kit with a lower limit of detection 0.1 unit/ml (Lonza Walkersville Inc., MD).

THP1 cells were grown in RPMI-1640 medium supplemented with glutamine, $10 \%$ heat inactivated Fetal Bovine Serum, $50 \mathrm{IU} / \mathrm{mL}$ Penicillin, $50 \mu \mathrm{g} / \mathrm{mL}$ Streptomycin, $10 \mathrm{mM}$ HEPES and $0.05 \mathrm{mM} \beta$-mercaptoethanol. Cells were treated with $1 \%$ Echinaforce ${ }^{\bullet}$ tincture versus ethanol solvent control. Each treatment condition consisted of six biological replicates. 


\section{Genome-wide gene expression analysis} Sample preparation and microarray processing

THP1 cells were treated for $48 \mathrm{~h}$ with $1 \%$ Echinaforce $^{\odot}$ or ethanol solvent control. RNA was isolated using the RNeasy mini kit (Qiagen) according to manufacturer's instructions. RNA concentration and purity was measured using the Nanodrop 1000 spectrophotometer (ThermoFischer, CA, USA). RNA integrity of each sample was checked using using the Experion Automated Electrophoresis System (Bio-Rad, MO, USA). Total RNA (500 ng) was amplified using the Illumina TotalPrep RNA Amplification kit (Life Technologies, Carlsbad, CA, USA). Briefly, RNA was reverse transcribed using T7 oligo (dT) primers, after which biotinylated complementary or anti-sense RNA (cRNA) was synthesized through an in vitro transcription reaction. Then, $750 \mathrm{ng}$ of amplified cRNA was hybridized to a HumanHT12 beadchip array (Illumina, San Diego, CA, USA) and further incubated for $18 \mathrm{~h}$ at $58^{\circ} \mathrm{C}$ in a hybridization oven under continuous rocking. After several consecutive washing steps, bead intensities were read on an Illumina iScan. Microarray data and raw gene expression intensities were preprocessed and analyzed using the beadarray $R$ package [30]. Intensities were quantile normalized and $\log _{2}$ transformed. Raw and normalized array data were uploaded to the Gene Expression Omnibus (GEO) database and have accession number: GSE117904. Probes with a P-detection value higher than 0.05 in at least six samples were removed. Also, probes annotated as "bad" and "no match" as described before [31] were not kept for further analysis. Differentially gene expression was performed using the limma $\mathrm{R}$ package [32]. P-values were corrected for multiple testing using the method of Benjamini and Hochberg. Probes with a log2 fold change higher than 0.4 and an adjusted $p$-value less than 0.05 were defined as significant and kept for further analysis [33]. The probes were annotated with gene information using the illuminaHumanv4.db annotation dataset [34]. The gene IDs of the significant Illumina expression probes were uploaded into the IPA software (Ingenuity ${ }^{\circ}$ Systems, www.ingenuity.com, Redwood City, CA, USA) to find enriched biological pathways, diseases and networks [35]. Fischer 's exact test was used to calculate a $p$-value determining the probability that each biological function and/or disease assigned to that data set is due to chance alone. Metascape systems biology freeware (https://metascape.org/) was used for correlating the transcriptomic profile data [36]. For each given gene list, pathway and process enrichment analysis has been carried out with the following ontology sources: KEGG Pathway, GO Biological Processes, Reactome Gene Sets, Canonical Pathways, CORUM, TRRUST, DisGeNET, PaGenBase, Transcription Factor Targets, WikiPathways, PANTHER Pathway and COVID. All genes in the genome have been used as the enrichment background. Terms with a $p$-value $<0.01$, a minimum count of 3 , and an enrichment factor $>1.5$ (the enrichment factor is the ratio between the observed counts and the counts expected by chance) are collected and grouped into clusters based on their membership similarities. More specifically, $p$-values are calculated based on the accumulative hypergeometric distribution, and q-values are calculated using the Banjamini-Hochberg procedure to account for multiple testings. Kappa scores are used as the similarity metric when performing hierachical clustering on the enriched terms, and sub-trees with a similarity of $>0.3$ are considered a cluster. The most statistically significant term within a cluster is chosen to represent the cluster. Heatmaps show Metascape enrichment analysis of all statistically enriched ontology terms (GO/KEGG terms, canonical pathways, hall mark gene sets). Accumulative hypergeometric $p$-values and enrichment factors are calculated and used for filtering. Remaining significant terms are then hierarchically clustered into a tree dendrogram based on Kappa-statistical similarities among their gene memberships. The term with the best $p$-value are selected within each cluster as a representative term to be displayed in a hierarchical tree dendrogram. The heatmap cells are colored by their $p$-values (see color legend). Along the same line, Metascape enrichment analysis of all statistically enriched TFtarget interaction networks is dermined by the TRRUST database [37]. Protein-protein interactions (PPI) among all input gene lists are extracted from PPI data source to form a PPI network (interactome). GO enrichment analysis is applied to the network to assign biological "meanings" of sub-protein networks. GO enrichment analysis is applied to each MCODE network to assign "meanings" to the network component, where top three best $p$-value terms were retained. MCODE components were identified from the merged network. Each MCODE network is assigned a unique color. For each given gene list, protein-protein interaction enrichment analysis has been carried out with the following databases: STRING, BioGrid, OmniPath, InWeb_IM. Only physical interactions in STRING (physical score $>0.132$ ) and BioGrid are used. The resultant network contains the subset of proteins that form physical interactions with at least one other member in the list. If the network contains between 3 and 500 proteins, the Molecular Complex Detection (MCODE) algorithm [38] has been applied to identify densely connected network components. The MCODE networks identified for individual gene lists have been gathered and are summarized in the MCODE subnetwork figure. Pathway and process enrichment analysis has been applied to each MCODE component independently, and the three best-scoring terms by $p$ value have been retained as the functional description of 
the corresponding MCODE components. Coronascape is a Metascape data hub including public available COVID-19 research related omics data sets. It includes more than 200 processed gene lists for SARS-CoV-2 retrieved from more than 20 published studies. These gene lists were generated using several omics technologies, including transcriptome (RNA-Seq and scRNASeq), proteome, phosphoproteome, ubiquitome, and interactome, providing a comprehensive picture of SARS-CoV-2 infection in various host cell and tissue types.

\section{Quantitative realtime PCR}

To validate microarray data, THP1 cells were treated with $1 \%$ Echinaforce ${ }^{\circ}$ or Solvent for the indicated timepoints $(3,6,12,24$ and $48 \mathrm{~h})$ in three independent experiments. The effect of JAK1 inhibition was determined by treating the cells with $1 \mu \mathrm{M}$ JAK1 inhibitor Filgotinib (GLPG0634, Selleckchem) for $30 \mathrm{~min}$ before adding Echinaforce ${ }^{\circ}$. Total RNA was isolated using the RNeasy mini kit (Qiagen, Hilden, Germany) including a DNAse treatment step as suggested by the manufacturer. Then 750 ng RNA was reverse transcribed into cDNA using oligo dT (Invitrogen), M-MLV reverse transcriptase (Promega, Wisconsin USA), $2.5 \mathrm{mM}$ dNTPs and RNaseOUT (Invitrogen). Samples were incubated on $42{ }^{\circ} \mathrm{C}$ for $60 \mathrm{~min}$ and $75^{\circ} \mathrm{C}$ for $15 \mathrm{~min}$. For the HERV genes, cDNA synthesis was performed using random primers (Invitrogen) and incubation of the samples at $37^{\circ} \mathrm{C}$ for $60 \mathrm{~min}$ and $75^{\circ} \mathrm{C}$ for $15 \mathrm{~min}$. qPCR was performed using the GoTaq qPCR Master Mix (Promega, Wisconsin USA) on a StepOnePlus Real-Time PCR machine (Applied Biosystems). Following primers were used: MX1 forward primer 5'-GTTTCCGAAGTGGACATCGCA3', MX1 reverse primer 5'-CTGCACAGGTTGTTCT CAGC-3' (NM_001144925), IFITM1 forward primer 5' CCAAGGTCCACCGTGATTAAC-3', IFITM1 reverse primer 5' -ACCAGTTCAAGAAGAGGGTGTT-3' (NM_003641), STAT1 forward primer 5' - CCATCCTT TGGTACAACATGC-3', STAT1 reverse primer 5'TGCACATGGTGGAGTCAGG-3' (NM_007315), IL8 forward primer 5'-GCTCTCTTGGCAGCCTTCCTGA3', IL8 reverse primer 5'-ACAATAATTTCTGTGTTG GCGC-3' (NM_000584), CXCL10 forward primer 5'GAAAGCAGTTAGCAAGGAAAGGT-3', CXLC10 reverse primer 5'-GACATATACTCCATGTAGGGAA GTGA-3' (NM_001565), ACTB forward primer 5'CTGGAACGGTGAAGGTGACA-3', and ACTB reverse primer 5' - AAGGGACTTCCTGTAACAATGCA-3' (NM_001101). Primer sequences for HERVs were derived from [39]. Each sample was ran in triplicate and the median $\mathrm{Ct}$-values between each replicate group was selected. Ct-values were normalized using ACTB housekeeping gene. The ddCt-values or log fold changes $(\log \mathrm{FC})$ were calculated using the solvent control as reference sample. A paired t-test t-test was used to determine the significance of the differences between Echinaforce ${ }^{\bullet}$ and solvent expression levels.

\section{Kinase activity profiling Sample preparation}

THP1 cells were treated with $1 \%$ Echinaforce ${ }^{ø}$ or ethanol solvent control for $15 \mathrm{~min}$. Cell lysates were prepared according to manufacturer's instructions. In short, cells were washed twice with cold 1X PBS and lysed with lysis buffer (1:100 dilution of Halt Phosphatase Inhibitor Cocktail and Halt Protease Inhibitor Cocktail EDTA free in M-PER Mammalian Extraction Buffer (ThermoFisher Scientific ${ }^{\mathrm{Tm}}$, Rockford, USA) at a ratio of $100 \mu$ l buffer per $1 \times 10^{6}$ cells. Lysates were then incubated on ice for 15 $\mathrm{min}$ and centrifuged for $15 \mathrm{~min}$ at $16000 \mathrm{xg}$ at $4{ }^{\circ} \mathrm{C}$. Protein concentration was quantified using the Pierce BCA Protein Assay Kit (ThermoFisher Scientific ${ }^{\text {mix }}$, Rockford, USA).

\section{Serine/threonine kinases (STK) and tyrosine kinase (PTK) pamgene assay and data analysis}

Kinase activity profiling was performed PamChip preprocessing and kinase activity profiling was performed according to manufacturer's instructions (PamGene International BV, 's-Hertogenbosch, The Netherlands). The first part of the protocol consisted in the blocking of the arrays with $2 \%$ BSA followed by several washing steps. Then $0.5 \mu \mathrm{g}$ for STK and $5 \mu \mathrm{g}$ for PTK assays together with the correspondent reaction mixes (purchased from the Pamgene) were loaded onto the arrays and incubated in the microarray system PamStation 12 instrument (PamGene International, Den Bosch, The Netherlands). In this step, the ATP contained in the mix leads to the activation of the kinases in the lysate which will result in the phosphorylation of the peptides on the array. Peptide phosphorylation intensities are then detected with the primary STK antibody mix and FITClabeled antibody for STK assay and with the FITClabelled PTK antibody (PTK assay). Images are then taken by the CCD camera in the PamStation ${ }^{\circ} 12$ and processed by the Bionavigator software. Peptide intensities data were $\log _{2}$ transformed and differences in phosphorylation between Echinaforce ${ }^{\circ}$ treated and control cultures were determined by using an univariate student $\mathrm{t}$-test analysis corrected for multiple testing using the Benjamini and Hochberg method [33].

To identify potentially activated or inhibited kinases we used the STK or PTK Upstream Kinase analysis PamApp from the Bionavigator Software. The analysis is based on "in silico predictions" for the upstream kinases of phosphorylation sites in the human proteome that are retrieved from the phosphoNET database [40]. In short, a prediction algorithm is derived from known 
interactions between kinases and phosphorylation sites. The prediction algorithm is then used to predict the strength of undocumented interactions. The Bionavigator application uses PhosphoNet database to map putative kinases upstream of the phospho-peptides (a kinase can have multiple possible phosphosites, and a single site can be phosphorylated by different kinases). For each set of peptides mapped to a specific kinase, a "difference statistics" is calculated (=normalized kinase statistics) using following formula: $\tau=\frac{1}{n} \sum_{i=1}^{n} \frac{\bar{p}_{i 1} \bar{p}_{i 2}}{\sqrt{s_{i 1}^{2}+s_{i 2}^{2}}}$ with $\bar{p}_{i j}$ and $\bar{s}_{i j}$ as the sample mean and variance of the intensity of peptide $i$ in group $j$, respectively, whereas $n$ is the number of peptides linked with a specific kinase. A positive kinase statistic means that the kinase is activated, while a negative statistic means the kinase is inactivated compared to the control group. The kinases are subsequently ranked based on a specificity and significance score which are calculated using permutation of the peptides and samples, respectively. Following formula is used: $Q=-\log _{10}$ $\left(\max \left(\frac{m}{M}, \frac{1}{M}\right)\right)$, where $\mathrm{m}$ is the number of times out of $\mathrm{M}$ permutations that $\left|\tau_{p}\right|>|\tau|$, where $\tau_{p}$ is the value of the difference statistic obtained after permutation of the samples or peptides. The significance score represents the magnitude of the change represented by the normalized kinase statistic. The specificity score represents the specificity of the of normalized kinase statistic in terms of the set of peptides used for the corresponding kinase. The higher the score the less likely it is that the observed normalized kinase statistics could have been obtained using a random set of peptides from the data set. The sum of the significance and specificity score is used to rank the kinases [41].

\section{Genome-wide DNA methylation analysis Sample preparation}

THP1 cells were cultured for $48 \mathrm{~h}$ with $1 \%$ Echinaforce $^{\circ}$ or ethanol solvent control. Corresponding cellular genomic DNA was isolated using the DNeasy Blood \& Tissue kit (Qiagen, Hilden, Germany) according to manufacturer's instructions. DNA concentration and purity was measured using the Nanodrop 100 spectrophotomer and $1 \mu \mathrm{g}$ of DNA was used for bisulfite conversion using the EZ DNA methylation Kit of Zymo Research according to manufacturer's instructions. Successful bisulfite conversion was checked using a methylation-specific PCR in a region of the SALL3 gene (see [42] for primer sequences).

\section{EPIC DNA methylation array}

The Infinium HumanMethylationEPIC BeadChip array (Illumina, San Diego, CA, USA) was used to measure genome-wide DNA methylation. Four $\mu \mathrm{L}$ of bisulfite-converted DNA from each sample was amplified, fragmented, precipitated, resuspended and subsequently hybridized onto the BeadChips. After overnight incubation of the BeadChips, unhybridized fragments were washed away, while hybridized fragments were extended using fluorescent nucleotide bases. Finally, the BeadChips were scanned using the Illumina iScan system to obtain raw methylation intensities of each probe.

\section{EPIC DNA methylation data preprocessing and analysis}

The $\mathrm{R}$ package RnBeads was used to preprocess the Illumina $450 \mathrm{~K}$ methylation data [43]. CpG-probes were filtered before normalization based on following criteria: probes containing a SNP within $3 \mathrm{bp}$ of the analyzed CpG site, bad quality probes based on an iterative greedycut algorithm with a detection $p$-value threshold of 0.01 , and probes with missing values in at least one sample. After filtering these CpG-probes, methylation values were within-array normalized using the beta mixture quantile dilation (BMIQ) method [44]. Another filtering step was performed after normalization based on following criteria: probes measuring methylation not at $\mathrm{CpG}$ sites (CC, CAG, $\mathrm{CAH}, \ldots$ ) and probes on sex chromosomes.

The methylation beta-values were transformed to M-values $\left(M=\log _{2}(\beta /(1-\beta))\right)$ prior to further analyses. The moderated $\mathrm{t}$-test incorporated in the limma $\mathrm{R}$ package [32] was used to calculate the statistics and $p$-values of the methylation differences between Echinaforce - and solvent-treated samples. Significant differentially methylated probes (DMPs) were selected based on a false discovery rate (FDR) < 0.1 and a difference in beta-value of at least 0.05 . The DMPs were annotated with gene information using the IlluminaHumanMethylationEPICmanifest $\mathrm{R}$ package [45]. Further gene information was retrieved from the UCSC genome browser (human hg19). Enrichment of genomic regions was calculated using the Fisher's exact test. Pathway analysis of the genes harboring a DMP was performed using the Ingenuity Pathway Analysis (IPA) software. Raw and normalized array data were uploaded to the Gene Expression Omnibus (GEO) database and have accession number: GSE117904.

\section{Protein expression of MX1, STAT1 and IFITM1 proteins using western blotting}

Protein expression levels of MX1, STAT1 and IFTI M1 were determined in THP1 cells treated with $1 \%$ Echinaforce $^{\circ}$ or ethanol solvent control for $48 \mathrm{~h}$, as explained before. Then, cells were washed and incubated $15 \mathrm{~min}$ on ice in lysis buffer containing: 150 $\mathrm{mM} \mathrm{NaCl}, 1 \mathrm{mM}$ EGTA, $1 \mathrm{mM}$ EDTA, $1 \mathrm{mM}$ ß- 
glycerolphosphate, $1 \%$ Triton X-100 (w/v), $20 \mathrm{mM}$ Tris $\mathrm{HCl}, \quad \mathrm{pH}=7.5$ and proteinase inhibitor (Complete $^{\mathrm{Tm}}$, EDTA-free Protease Inhibitor Cocktail, Sigma-Aldrich, USA) plus PhosphataseArrest ${ }^{\text {th }}$ Phosphatase Inhibitor Cocktail (phosphataseArrest ${ }^{\mathrm{Tm}}$, GBiosciences, USA). Cells were subsequently centrifuged for $15 \mathrm{~min}$ at $200 \mathrm{~g}$ at $4{ }^{\circ} \mathrm{C}$ and supernatant containing the soluble proteins were stored at $-20^{\circ} \mathrm{C}$ until use. Protein lysates $(20 \mu \mathrm{g})$ were mixed with $5 \mathrm{X}$ sample buffer (5\% SDS, $20 \%$ glycerol, $0.2 \%$ bromophenol-blue, $250 \mathrm{mM}$ DTT, $65 \mathrm{mM}$ Tris $\mathrm{HCl}$ ) all purchased from Sigma Aldrich (Missouri, USA), heated for $5 \mathrm{~min}$ at $95^{\circ} \mathrm{C}$ and loaded in a $12 \%$ SDSPAGE gel. Proteins contained in the homogenates were separated during $30 \mathrm{~min}$ at $60-70 \mathrm{~V}$ and $1 \mathrm{~h}$ at a constant voltage of $130 \mathrm{~V}$. Further, $10 \mu \mathrm{l}$ of BenchMark $^{\text {Tat }}$ Pre-Stained Protein Standard (Life Technologies, CA, USA) was also loaded next to the samples. After separation proteins ttransferred onto a Nitrocellulose Membrane (BioRad, CA, USA) during $2 \mathrm{~h}$ at $45 \mathrm{~V}$. Non-specific binding sites were blocked by incubating the membranes with blocking buffer $(0.05 \%$ Tween 20, 1x TBS, 5\% BSA) for $1 \mathrm{~h}$ at room temperature. The membrane was then incubated with the primary antibodies: MX1 (D3W7I) Rabbit mAb \#37849, IFITM1 Antibody Rabbit pAb \#13126 and the STAT1 (42H3) Rabbit mAb \#9175 (all purchased from Cell Signaling Technology, Massachusetts, USA) or rabbit polyclonal Anti-GAPDH antibody (ab9485, Abcam, Cambridge, UK) overnight at $4{ }^{\circ} \mathrm{C}$. After membranes were washed, they were incubated with (1:10000) Donkey anti-Rabbit IgG $(\mathrm{H}+\mathrm{L})$ Secondary Antibody-HRP (Thermo Fisher Scientific, Massachusetts, USA) for $1 \mathrm{~h}$ at room temperature. Chemiluminiscence detection was performed using the ECL detection kit (Pierce ${ }^{\mathrm{Ta}}$ ECL Western Blotting Substrate (Thermo Fisher Scientific, Massachusetts, USA) in a ChemiDoc MP system (BioRad, CA, USA).

\section{Assessment of IFNa2, IFN $\beta$ IFN $\gamma$, CXCL8 (IL8) and CXCL10 levels}

Cell culture supernatants were collected after 3, 6, 12, 24 and $48 \mathrm{~h}$ and assayed for chemokines CXCL10 and IL8 by means of an enzyme-linked immunosorbent assay (ELISA) purchased from Invitrogen (CA, USA) following manufacturer's instructions. The assays have a detection limit of $2 \mathrm{pg} / \mathrm{ml}$ for CXCL10 and $5 \mathrm{pg} / \mathrm{mL}$ for IL-8. Similarly, protein concentrations of IFN $\alpha 2$, IFN $\beta$ and IFN $\gamma$ were measured in the same culture supernatants using the highly sensitive U-PLEX Biomarker Group 1 (hu) Assay (Meso Scale Diagnostics, Maryland, USA) following manufacturer's instructions. The U-PLEX assays have a detection limit of $4.0 \mathrm{pg} / \mathrm{ml}, 3.1 \mathrm{pg} / \mathrm{mL}$ and 1.7 $\mathrm{pg} / \mathrm{mL}$ respectively for IFN $\alpha 2$, IFN $\beta$ and IFN $\gamma$.

\section{Results}

Echinaforce ${ }^{\circledR}$ treatment triggers tonic IFN regulation of innate immunity signaling pathways

Widespread gene expression changes in monocyte THP1 cells were detected upon $48 \mathrm{~h} 1 \%$ Echinaforce ${ }^{\circ}$ treatment. Based on significance criteria of FDR $<0.05$ and absolute $\log _{2}$ fold change $>0.4$, Echinaforce ${ }^{\bullet}$ induced modest upregulation of 205 expression probes (173 genes) while 124 probes (99 genes) were downregulated compared with the ethanol treated solvent controls (Fig. 1a and Supplementary Table 1). In contrast to pharmacological drugs (for example glucocorticoids (GC)) which can trigger drastic expression changes of GC-responsive genes (typically, $\log _{2}$ fold $>1$ ), many bioactive phytochemicals rather induce moderate transcriptional changes (typically $\log _{2}$ fold $>0,4$ ) of multiple genes converging on the same pathway [46-48]. Genes differentially expressed (DEG) by Echinaforce ${ }^{\bullet}$ treatment were enriched for IPA canonical pathways related to innate immune responses including interferon signaling, interferon regulatory factor (IRF) activation and the role of pattern recognition receptors, among others (Fig. 1bc and Supplementary Table 2). Interestingly, most of these pathways were predicted to be activated, as can be seen from the highly positive activation z-scores. Interferon (IFN) $\alpha / \beta$ and IFN $\gamma$ both induce IFN-stimulated gene (ISG) expression through Janus kinase (JAK)dependent phosphorylation of signal transducer and activator of transcription factors (STAT) 1 and STAT2 [49-54]. In line with the latter reports, we could observe transcriptional activation of various antiviral gatekeepers and interferon inducible proteins (i.e. MX1, IFI6/27/35/ 44, IFITM1/2/3, IFIT1/2/3, ISG15/20, IRF7/9), including various STAT1 target genes (Fig. 1c, Supplementary Table 1, 2). Logically, pathways related to viral infection and replication were predicted to be inhibited (activation $\mathrm{z}$-score $<-2$ ). Also pathways involving cellular movement, migration, recruitment and chemotaxis were predicted to be activated (activation z-scores $>2$ ) (Fig. 1d). Aside from ISGs, transcription of various chemokines and receptors (CXCL10, CXCL8, CCL2, CCL5, and CXCR4) were also increased. In full accordance, recruitment and adhesion of immune cells, infection and immune related processes were found top ranked enriched diseases and biological functions in IPA analysis (Supplementary Table 3).

Complementary to IPA analysis, protein-proteininteraction enrichment analysis of DEGs by STRING [55] and Metascape [56] algorithms was performed. This revealed strong enrichment of protein-protein interactions responding to a chemical stimulus, which triggers a defensive antiviral innate immune response involving IFN, TLR, NOD, RIG, cytokine, chemokine and NFKB signaling pathways (Fig. 2, Supplementary Table 4). 


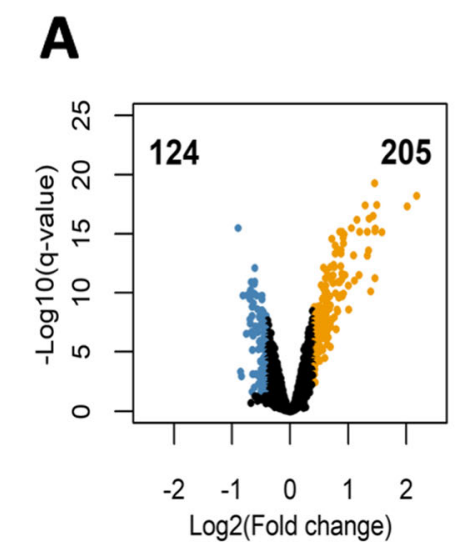

B

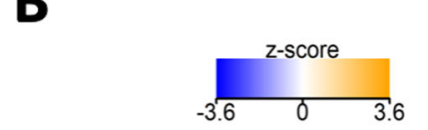

\begin{tabular}{lllll}
\multicolumn{4}{c}{$-\log (\mathrm{P}$-value $)$} \\
2 & 4 & 6 & 8 & 11
\end{tabular}

\section{ШШШШШ}

Activation of IRF by Cytosolic Patert Recognition Receptors Death Receptor Signaling Atherosclerosis Signaling Dendritic Cell Maturation Granulocyte Adhesion and Diapedesis Agranulocyte Adhesion and Diapedesis Hepatic Fibrosis / Hepatic Stellate Cell Activation Communication between Innate and Adaptive Immune Cells Role of Pattern Recognition Receptors in Recognition of Bacteria and Viruses Role of PKR in Interferon Induction and Antiviral Response Role of Hypercytokinemia/hyperchemokinemia in the Pathogenesis of Influenza
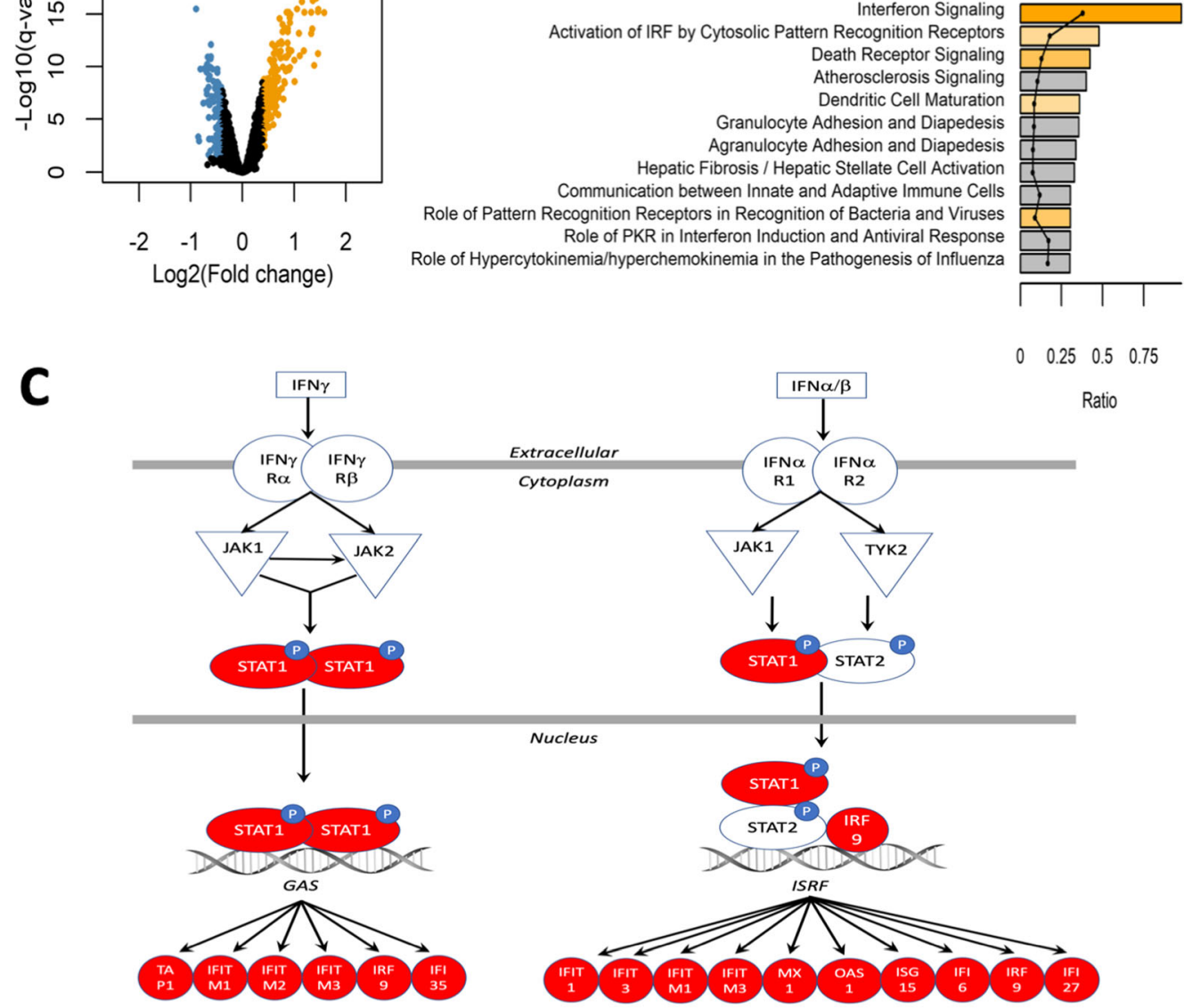

D
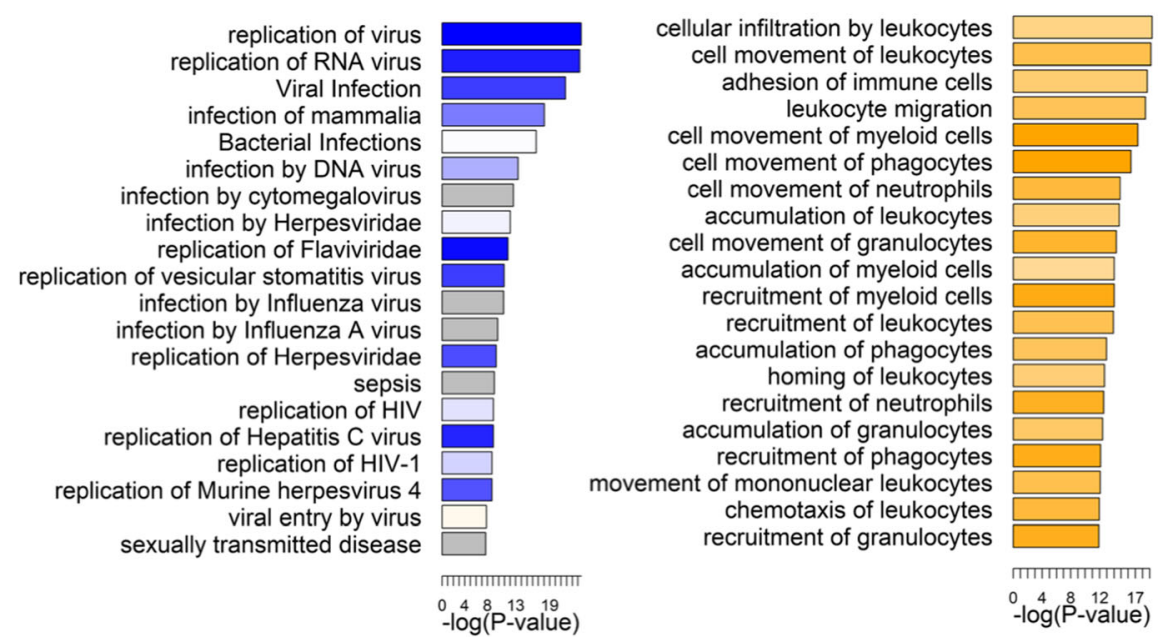

Fig. 1 (See legend on next page.) 
(See figure on previous page.)

Fig. 1 Echinaforce ${ }^{\oplus}$ induced gene expression activates innate immunity pathways a Volcano plot showing the upregulated genes (orange color, number of probes: 205), and downregulated genes (blue color, number of probes: 124) upon treatment of THP1 cells for $48 \mathrm{~h}$ with Echinaforce ${ }^{\oplus}$ tincture (1\%). b Top enriched IPA canonical pathways. Bars are colored by activation z-score. c IPA interferon signaling pathway with Echinaforce ${ }^{\oplus}$-induced upregulated genes colored in red and green, respectively. $\mathbf{d}$ Top enriched IPA infectious diseases and IPA immune trafficking disease and biological function. Bar charts are colored by activation z-score

More particularly, Metascape MCODE analysis identified 3 interconnected subnetworks in the antiviral cytokine response: cellular response to interferon, regulation of leukocyte chemotaxis and (mitochondrial) metabolism (Supplementary Table 4).

Next, different gene members of the IFN and chemotaxis innate immune signaling pathway, responsive to Echinaforce $^{\circ}$ treatment in THP1 cells (Fig. 3a) were selected for further evaluation of time dependent expression changes: STAT1, MX1, IFITM1, IFN 2 2, IFN $\beta$, IFNy, CXCL8 and CXCL10 mRNA and/or protein levels were measured in THP1 monocytes after 3 to $48 \mathrm{~h}$ Echinaforce ${ }^{\circ}$ treatment by means of qPCR, ELISA, multiplex MSD U-PLEX ${ }^{\bullet}$ immunoassay and/or Western immunoblotting assays. Induction of STAT1 and the interferonstimulated genes MX1 and IFITM1 expression could

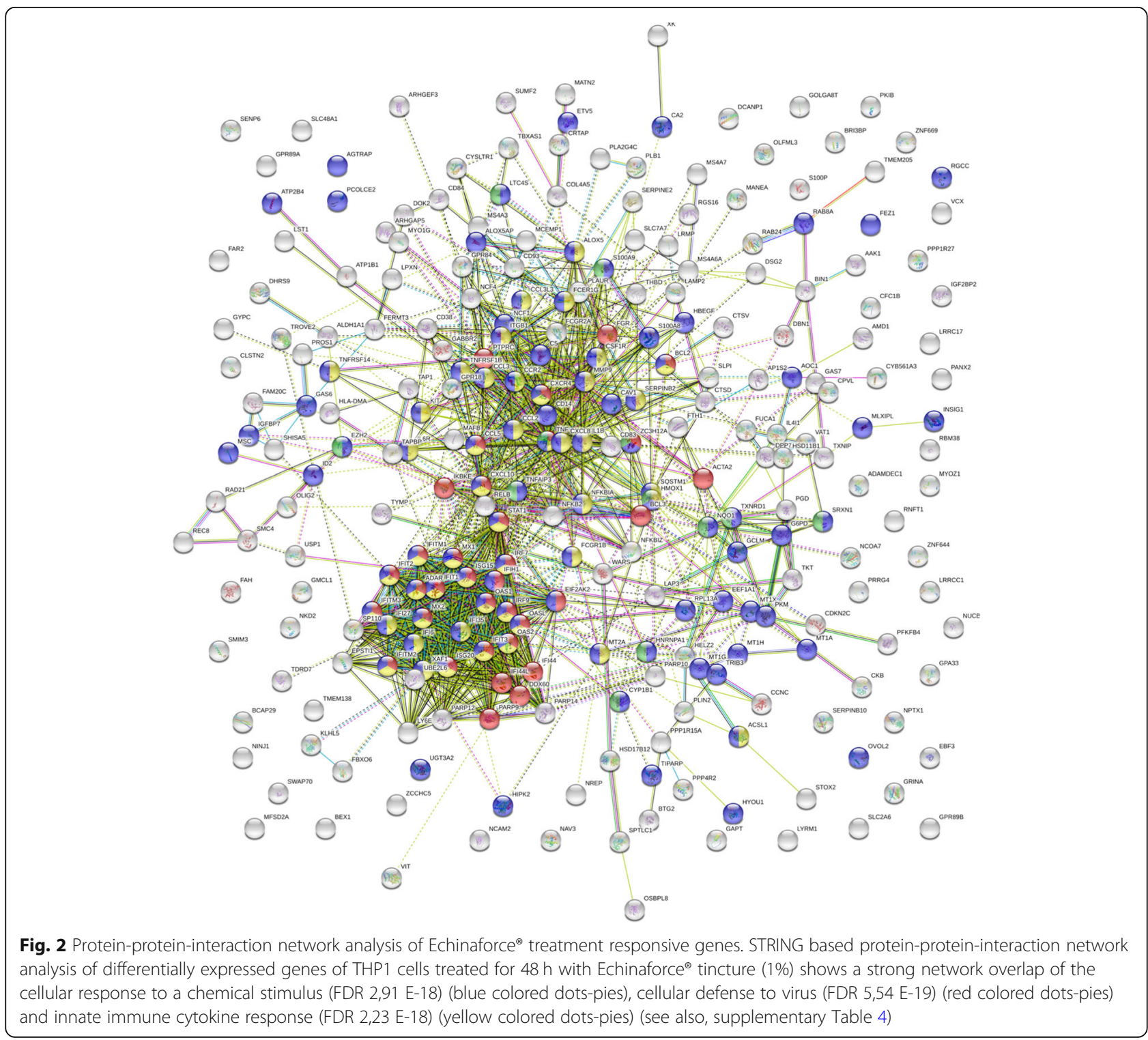



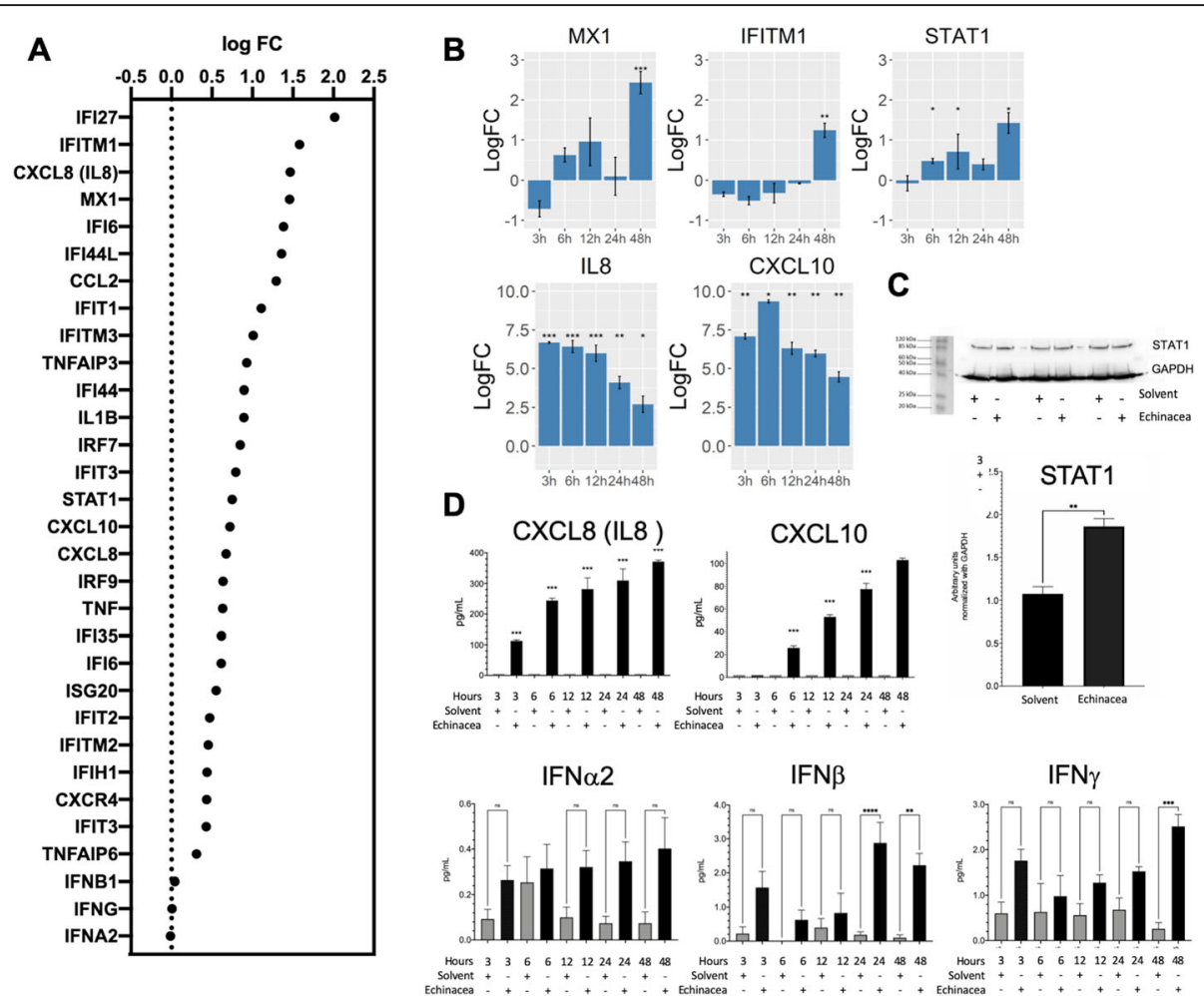

Fig. 3 Induction of innate immune response by Echinaforce ${ }^{\oplus}$. A) transcriptome gene expression changes IFN, innate immunity, chemokine, cytokine genes (logFC) B) transcription levels of MX1, IFITM1, STAT1, CXCL8(IL8) and CXCL10 genes at different time points, the bars represent the mean logFC values +- SD compared to the solvent control. ${ }^{*}: P \leq 0.05,{ }^{* *} P: \leq 0.01,{ }^{* * *} P: \leq 0.001$ and ${ }^{* * * *} P: \leq 0.0001$. C) Blots showing protein levels of STAT1 and GAPDH (as reference protein) in $20 \mu \mathrm{g}$ protein of cell lysates after $48 \mathrm{~h}$ stimulation with solvent (Ethanol) or Echinaforce ${ }^{\circledast}$; Bars graph represents the density of each blot band for STAT-1 relative to the band density of GAPDH (reference protein). Band intensities were calculated using imageJ software. Statistical differences between solvent and Echinacea treated samples were assayed using a paired t-test where $p$ value $<0.05$ was considered statistically significant. $\left({ }^{* *}\right)$ means $p$ value $<0.01,\left({ }^{*}\right): P \leq 0.05,\left({ }^{* *}\right): P \leq 0.01,\left(*^{* *}\right): P \leq 0.001$ and $\left.\left({ }^{* * *}\right): P \leq 0.0001 . D\right)$ Expression levels of IL8, CXCL10, IFNa2, IFN 3 , IFNy chemokines assayed by ELISA and MSD-U-Plex immunoassays in supernatants collected after Echinaforce ${ }^{\oplus}$ and solvent (Ethanol) stimulation. $\left(^{* *}\right)$ means $p$ value $<0.01,\left({ }^{*}\right): P \leq 0.05,\left({ }^{* *}\right): P \leq 0.01,\left({ }^{* *}\right): P \leq 0.001$ and $\left({ }^{* * *}\right): P \leq 0.0001, P$-values after a paired t-test where $p$ value $<0.05$ was considered statistically significant

clearly be confirmed, with maximal mRNA transcription levels observed after $48 \mathrm{~h}$ treatment (Fig. 3b). Corresponding changes in STAT1 protein expression levels could also be verified by Western analysis (Fig. 3c), whereas antibodies failed to detect significant amounts of MX1 and IFITM1 protein (data not shown). Whether MX1 and IFITM1 protein expression has high turnover rates resulting in low protein expression levels needs further investigation $[57,58]$. For the chemokines IL8 and CXCL10, persistent gene induction could be observed upon Echinaforce treatment until $48 \mathrm{~h}$, with peak transcription levels after $3 \mathrm{~h}$ (Fig. 3b). Accordingly, time dependent accumulation of both chemokines in the cell culture supernatants could be detected in ELISA (Fig. 3d). Finally, in line with background mRNA transcription levels, multiplex immunoassay detection of supernatant levels of IFN $\alpha 2$, IFN $\beta$, IFN $\gamma$ protein only showed low expression levels, which weakly increase after $48 \mathrm{~h}$ Echinaforce ${ }^{\bullet}$ treatment Fig. 3e). However, in contrast to high expression levels of IFN upon acute viral infection, very weak expression levels of IFN in absence of infection also exert profound immunological effects, in part through "tonic" homeostatic modulation of various signaling intermediates which regulate diverse cytokines to train immunity [59-61].

\section{Echinaforce ${ }^{\circledast}$ treatment activates IFN and antiviral innate immune response which is suppressed in severe SARS- CoV-2 patients}

Coupled to Metascape analysis [36], the Coronascape database (https://metascape.org/COVID) provides quick access to numerous published COVID-19 omics data sets, and a comprehensive system level data analysis toolkit for data mining. Remarkably, upon comparison of our Echinaforce ${ }^{\circ}$ responsive gene signature in THP1 monocytes with public available datasets of gene expression profiles of SARS-CoV2 patients, we observed a very strong overlap in enriched pathways ( $P$-value $10^{-48}$ $10^{-61}$ ) related to IFN, cytokine and innate immune signaling in patients with mild to severe symptoms [62-69] 
(Fig. 4a). Of special note, whereas Echinaforce ${ }^{\bullet}$ treatment was found to promote innate immunity via multiple IFN stimulated genes (ISG), i.e. pattern recognition receptor genes and chemokines (our results), severe SARS-Cov2 patients typically suffer from a strongly impaired interferon (IFN) type I response and weak innate antiviral defense (ISGs), associated with a persistent blood viral load and an exacerbated inflammatory response [64-72]. Furthermore, Metascape TRRUST analysis [37] of all statistically enriched TF binding motifs in differentially expressed genes in severe covid patients, which can be modulated by Echinaforce treatment identified key roles for NFkB, STAT and IRF family transcription factors (Fig. 4b). Finally, Metascape Proteinprotein interaction analysis of Echinaforce regulated protein networks identified multiple antiviral IFN and immune signaling networks disturbed in severe SARS-CoV2 patients (Fig. 4c, Supplementary Table 4), including an EBV specific virus infection protein network. Remarkably, EBV reactivation and increased EBV DNA load have recently been reported in severe SARS-CoV2 patients with impaired lymphocyte subpopulation counts [73].

\section{Echinaforce ${ }^{\circledR}$ treatment activates JAK1, NFKB and MAPK kinases}

To identify most important upstream kinase pathways responsible for gene expression changes in THP1 monocytes following Echinaforce ${ }^{\circledR}$ treatment, we performed a Pamchip kinome activity profiling assay [41]. This peptide array approach allows characterization of cellular serine/threonine or tyrosine kinome activity profiles following on chip in vitro kinase reaction of 144 conserved kinase consensus peptide motifs in presence of THP1 monocyte lysates left untreated or following Echinaforce ${ }^{\bullet}$ treatment [74-77]. Using the upstream kinase prediction tool of the Bionavigator PamGene software, the qualitative and quantitative changes in phosphopeptide chip intensities upon Echinaforce ${ }^{\bullet}$ treatment were translated into a pattern of activated or inhibited upstream kinases (Fig. 5a and Supplementary Table 5). In agreement with the transcriptional activation of the IFN signaling pathway described above (Fig. 1c), Pamchip kinome profiling [41] revealed activation of the JAK1 kinase which is important in the phosphorylation of STAT kinases and subsequently downstream regulation of IFN-stimulated genes. Furthermore, in line with pathway analysis of transcriptome data, we also identified activation of the tyrosine kinase TEC (Fig. 5b) (Supplementary Table 2, 3, 4). Surprisingly, our analysis did not detect significant activity changes of early IFN kinases TBK1 and IKK [78].

Besides, we also identified various Echinaforce ${ }^{\oplus}$ activated kinases belonging to the MAPK superfamily of

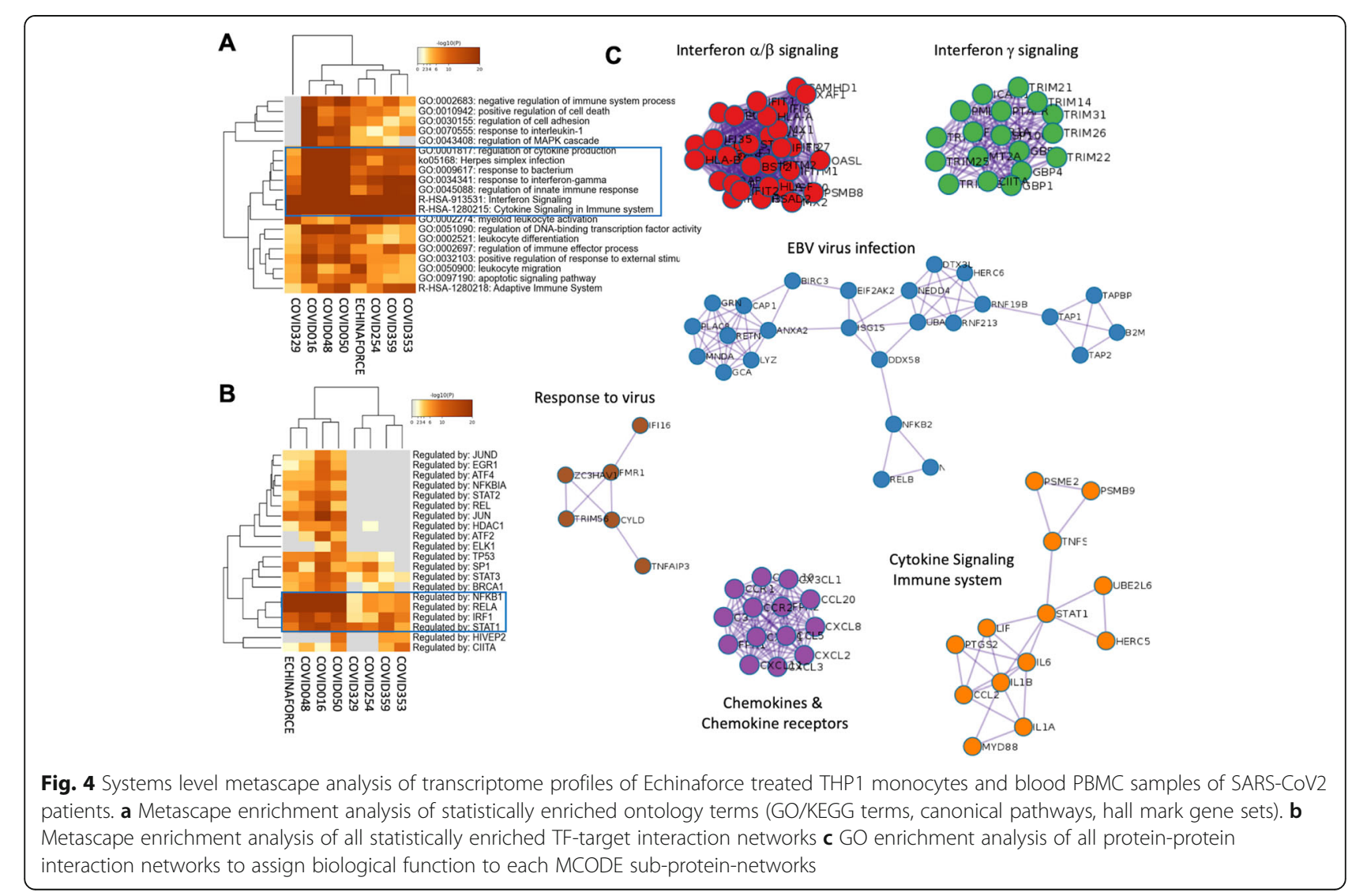



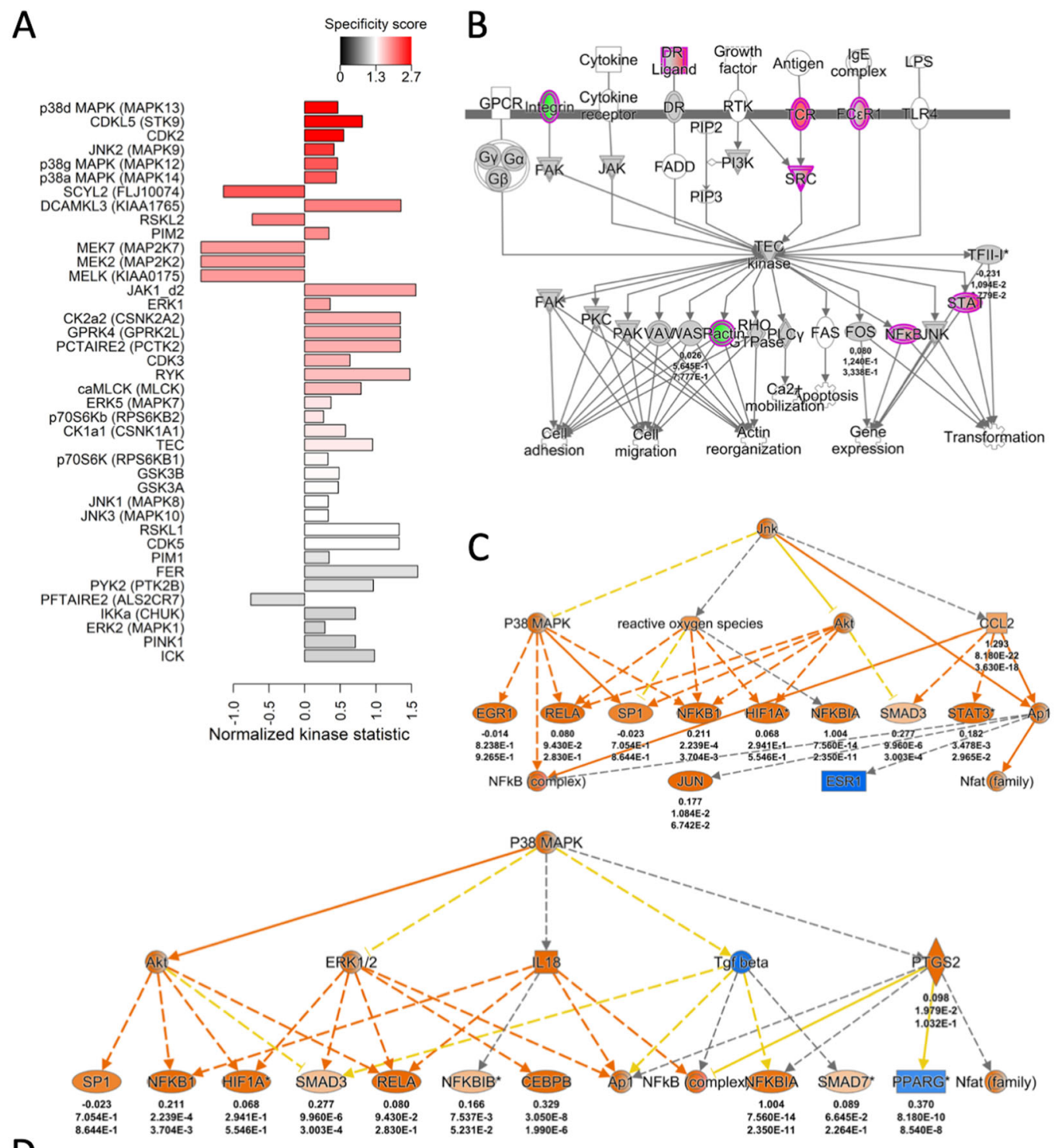

D

\section{CXCL10}

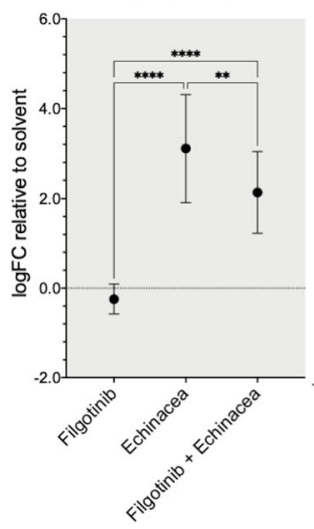

IFITM1

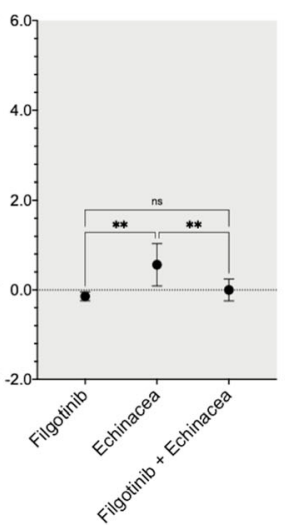

CXCL8 (IL8)

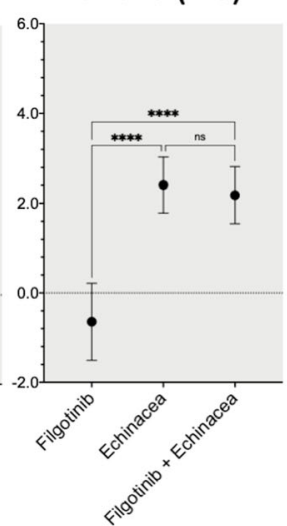

MX1

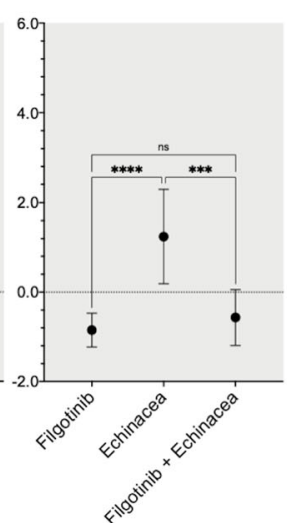

STAT1

Fig. 5 (See legend on next page.)

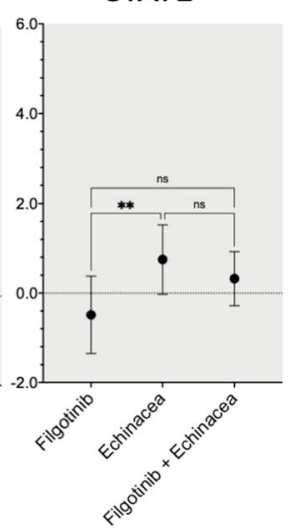




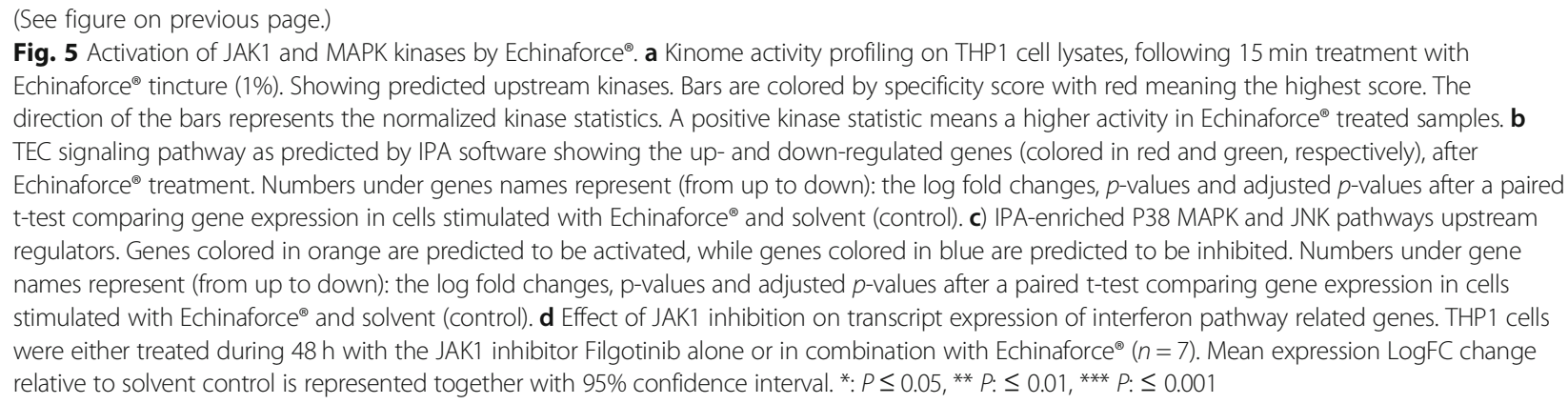

kinases: p38 MAPK (MAPK11, - 12, - 13, and - 14), JNK (MAPK8, -9 and -10 ) and ERK1 (Fig. 5c). This upstream regulators are also predicted by IPA to control various canonical pathways, including pattern recognition receptors in recognition of bacteria and viruses, activation of IRF by cytosolic pattern recognition receptors and role of MAPK signaling in the pathogenesis of influenza among others.

To further verify crucial involvement of JAK kinase activation in downstream gene expression effects upon Echinaforce ${ }^{\odot}$ treatment, we compared THP1 gene expression changes following Echinaforce ${ }^{\oplus}$ treatment in presence or absence of the pharmacological JAK1 inhibitor filgotinib. We found that filgotinib significantly suppresses the Echinaforce ${ }^{\circ}$ responsive genes $\mathrm{MX} 1$ and IFITM1, whereas STAT1, CXCL10 and IL8 gene expression were less significantly suppressed (Fig. 5d). Altogether, experiments with the JAK1 inhibitor filgotinib strenghten our transcriptome and kinome data analysis, pointing to JAK1specific regulation of downstream gene expression changes in response to Echinaforce ${ }^{\oplus}$ treatment.

\section{Echinaforce ${ }^{\circledast}$ treatment elicits epigenetic changes in innate immunity gene pathways}

Epigenetics seems to be important in training immunity $[60,79]$ during monocyte differentiation and in the immunological memory of macrophages [80, 81]. Today, various bioactive phytochemicals have been identified which modulate inflammation through epigenetic reprogramming [82, 83]. Different phytochemicals and nutrients are known to change DNA methylation and histone modifications by directly influencing epigenetic enzymes or by interfering with the availability of the substrates/ cofactors of these enzymes [84-86]. To assess whether the Echinaforce ${ }^{\oplus}$ induced changes in transcriptome profiles in THP1 cells are associated with DNA methylation changes, we measured complementary changes in DNA methylation profiles using the Illumina EPIC methylation array. Significant DNA methylation changes were observed following $48 \mathrm{~h}$ exposure to Echinaforce ${ }^{\odot}$ (Fig. 6a and Supplementary Table 6).
A total of $1875 \mathrm{CpG}$ sites was found differentially methylated (FDR $<0.1$ ) with a methylation difference of at least 5\%. Typically, DNA methylation changes after short (24-72 h) exposure to phytochemicals and nutrients are much smaller than cancer associated DNA methylation changes in oncogenes or tumor suppressor genes which accumulate for many years in response to the microenvironment $[48,87,88]$. However, similar DMR effects sizes and cutoff $(<5 \%)$ were found to be biologically meaningful in various disease etiologies [42, 89, 90].

From the 1875 CpG sites identified, only 40 differentially methylated positions (DMPs) were hypomethylated whereas 1835 DMPs were hypermethylated. DMPs were mainly enriched in gene bodies, intergenic, and CpGpoor regions, while depleted in CpG islands, promoter, and enhancer regions (Fig. 6b). Only 1259 of the 1875 CpG-probes (67\%) were located in a gene or 1500 bp upstream of a gene. Similarly, DNA methylation variation in the immune system was predominantly found at at CpG islands (CGI) within gene bodies, which have the properties of cell type-restricted promoters, but infrequently at annotated gene promoters or CGI flanking sequences (CGI "shores") [91]. Subsequent IPA pathway enrichment analysis of the genes containing DMPs revealed inflammation or immunological diseases among others (Supplementary Table 6). Of particular interest, one of the top enriched pathways ('Superpathway of Inositol Phosphate Compounds') controls various epigenetic processes related to the interferon response [92-94].

Since both gene expression and kinase profiling both revealed the involvement of interferon signaling pathways, we also checked whether methylation of IFN pathway genes was affected by Echinaforce ${ }^{\bullet}$ treatment. Eight probes located in BCL2, JAK1, STAT1, PIAS1 and TAP1 did show an FDR $<0.1$, with small methylation differences (between 1 and 3\%) (Fig. 6c). Whether these small methylation changes are sufficient to "train" the innate immune gene response needs further investigation [60, 61, 79].

Since most of the DMPs were located in intergenic regions and gene bodies, only a small subset of genes 

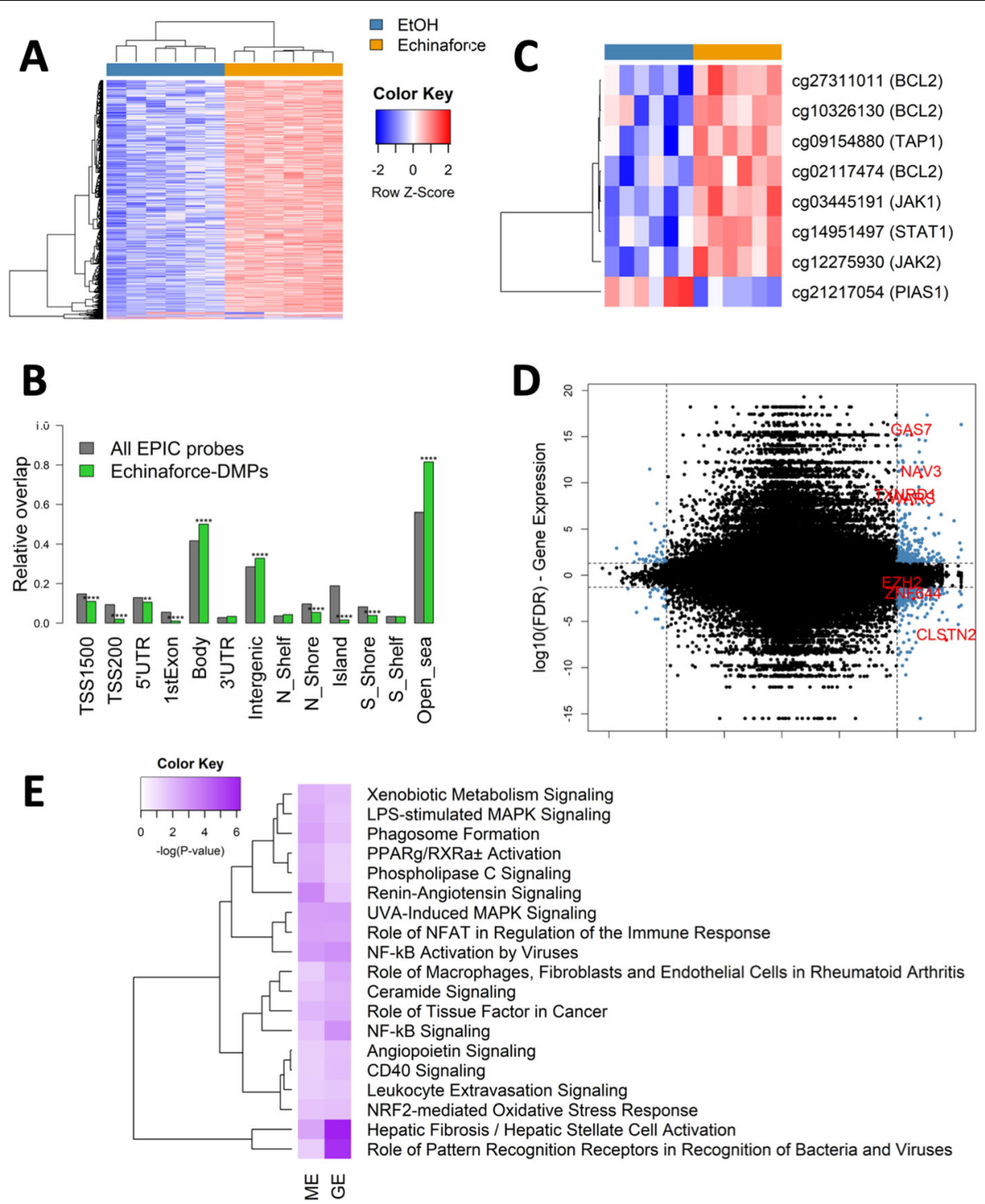

Fig. 6 Echinaforce ${ }^{\oplus}$ treatment leads to global hypermethylation of CpG-poor gene bodies. a Heatmap showing the methylation values of differentially methylated probes upon treatment of THP1 cells for $48 \mathrm{~h}$ with Echinaforce ${ }^{\circledast}$ tincture (1\%). Solvent (EtOH) controls are colored in blue and Echinaforce ${ }^{\oplus}$-treated cells in orange. $\mathbf{b}$ Genomic enrichment of DMPs in different genomic regions. c CpG probes located in genes of the interferon signaling pathway which were differentially methylated (FDR $<0.1)$. $P \leq 0.05,{ }^{* *} P \leq 0.01,{ }^{* * *} P \leq 0.001,{ }^{* * * *} P \leq 0.0001$. d Starburst plot showing the genes both differentially expressed and differentially methylated. Each CpG-probe was mapped to its corresponding gene and the $-\log 10(F D R)$ from the gene expression and DNA methylation analysis is displayed. The -log10(FDR) values of genes or CpG-probes with a negative LogFC or delta beta was multiplied by -1 leading to positive values when logFC or delta beta was positive and negative values when logFC or delta beta was negative. CpG-probe - gene pairs which were differentially expressed $(F D R<0.05)$ and differentially methylated (FDR $<$ 0.1) were colored in blue. The CpG-probe - gene pairs of which the absolute delta beta was higher than 0.05 and the absolute logFC higher than 0.4 were colored in red. e The IPA canonical pathways which were both significantly enriched in the gene expression and DNA methylation analysis

containing a DMP also resulted in a significant change in gene expression (Fig. 6d). Only seven genes were both differentially methylated and expressed, based on the significance criteria described above: i.e. Calsyntenin 2 (CLSTN2), Enhancer Of Zeste 2 Polycomb Repressive Complex 2 Subunit (EZH2), Growth arrest-specific protein (GAS)-7, neuron navigator (NAV)-3, Thioredoxin
Reductase (TXNRD)-1, Tryptophanyl-tRNA synthetase (WARS) and Zinc Finger Transcription Factor (ZNF)644. When using less stringent significance criteria, leaving out the effect size cutoff $(\operatorname{logFC}), 574 \mathrm{CpG}$ site gene pairs were found to be differentially expressed and methylated. Upon further comparing canonical pathways which are significantly enriched for both lists of 
differentially expressed genes and the list of differentially methylated genes, we identified 10 common biological processes (Fig. 6e). Remarkably, common pathways include NF- $\kappa B$ signaling (NF- $\kappa B$ activation by viruses, NF$\kappa B$ signaling), MAPK signaling (LPS-stimulated MAPK signaling, UVA-induced MAPK signaling), and immune responses (i.e. Role of pattern recognition receptors in recognition of bacteria and viruses, Role of NFAT in regulation of the immune response, phagosome formation, CD40 signaling, leukocyte extravasation signaling).

\section{Echinaforce ${ }^{\circledast}$ treatment changes DNA repeat methylation and HERV transcription levels}

DNA repeats and transposons require hypermethylation to maintain genomic instability and prevent transposition [95-99]. Interestingly, differentially methylated probes (DMPs) demonstrated a considerable enrichment in LINE, SINE and LTR transposon repeats, flanking endogenous retroviral sequences (HERVs) (Fig. 7a-b). This DMPs decreased transcription of MER4D, MER57B1, MLT1C627, MLT2B4 HERVs after 12 and $48 \mathrm{~h}$ Echinaforce $^{\circ}$ treatment, whereas MLT1B and MLT1C49 HERVs were only transiently repressed at $12 \mathrm{~h}$ (Fig. 7c). However, it remains unclear whether innate immune signaling (IFN response, chemotaxis, and immunometabolism) is driving HERV regulation or vice versa to mediate viral protection.

\section{Discussion}

In this study, we applied for the first time a systems biology approach to characterize a possible mode of action of a standardized medicinal Echinacea purpurea (L.) Moench tincture Echinaforce ${ }^{\circ}$, which is widely used as a herbal remedy against respiratory tract infections. Microarray, QPCR, Western and multiplex immunoassays demonstrate that treatment of THP1 monocyte cells with Echinaforce ${ }^{\bullet}$ phytochemicals elicit time dependent gene expression changes in antiviral innate immunity signaling networks, involving tonic IFN (MX1, IFN $\beta$, IFNy, IFITM1, STAT1, STAT2) chemotaxis (IL8, CXCL10) and immunometabolic (ISG15, PKM2, SQST M1) signaling pathways.

Most cells express a set of membrane and cytoplasmic receptors to detect viral RNA and DNA molecules: Pattern Recognition Receptors (PRRs). These receptors control innate immune signaling to activate the synthesis of interferons during a viral infection. In addition to pathogens, autophagy, metabolic and chemical stress, DNA damage, unfolded protein response, can also regulate innate immunity through cell-autonomous responses. Either IFN-inducible or constitutive, these processes aim
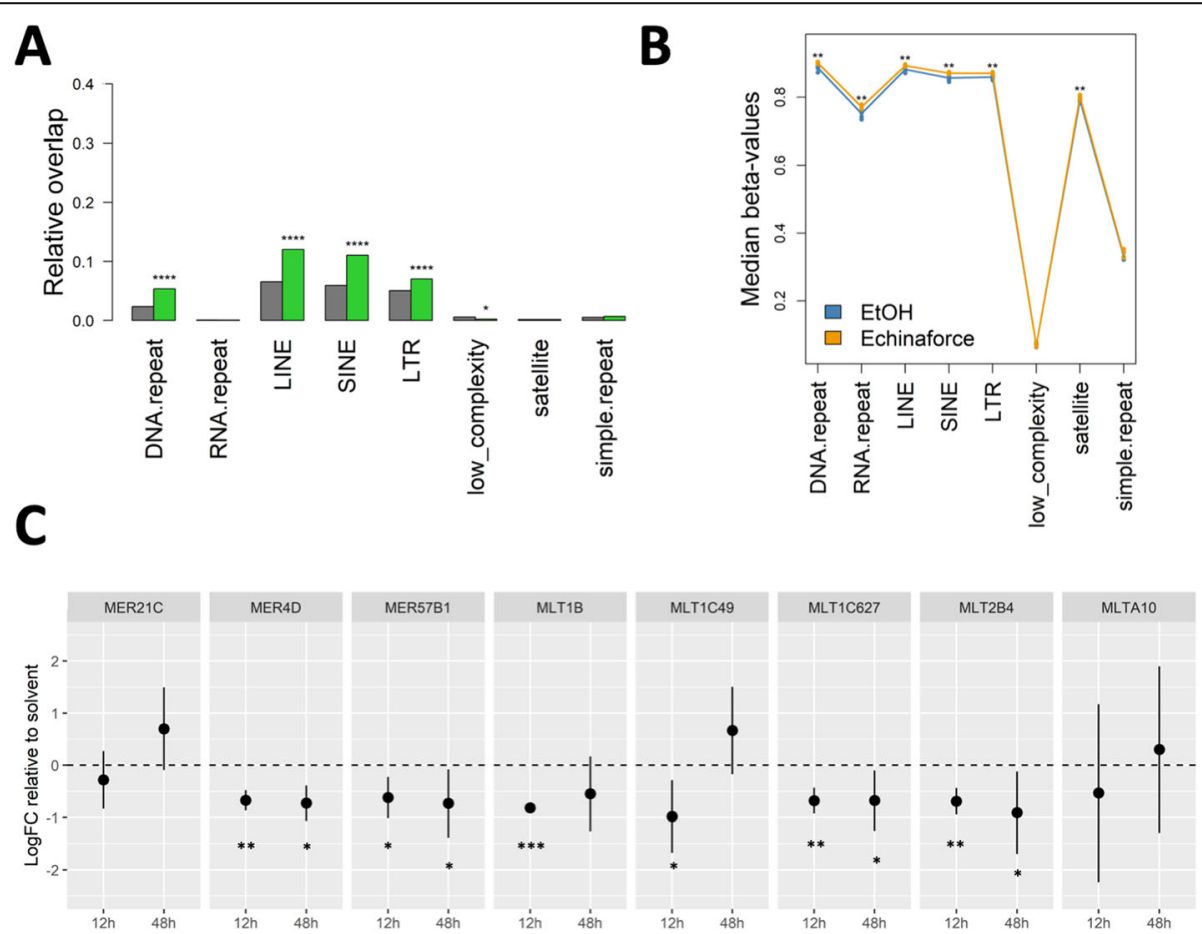

Fig. 7 Echinaforce ${ }^{\oplus}$ treatment leads to global hypermethylation of intergenic repeat elements. a Genomic enrichment of DMPs in different repeat elements. $\mathbf{b}$ Global DNA methylation changes in different repeat elements. $\mathbf{c}$ HERV qPCR gene expression. THP1 cells were with Echinaforce ${ }^{\circledR}$ at 12 and $48 \mathrm{~h}(n=3)$. Mean LogFC change relative to solvent control is represented together with $95 \%$ confidence interval. ${ }^{*}: P \leq 0.05,{ }^{* *} P: \leq 0.01$, *** $P: \leq 0.001$ and ${ }^{* * *} P: \leq 0.0001$ 
to guarantee cell homeostasis or a biodefense mechanism against (non-self) hazardous molecules [100]. Of importance, these distinct constitutive cell-autonomous responses appear to be interconnected and can also be modulated by microbes, viruses and PRRs [101]. Our results suggest that Echinaforce ${ }^{\circ}$ phytochemicals train innate immunity pathways via activation of interferon and chemokine gene expression. As such, secondary metabolite phytochemicals involved in plant immunity may prime evolutionary conserved innate immune responses across species [102-104].

For example, Echinaforce ${ }^{\ominus}$ treatment increases expression IFI27 and IFITM1, which both play critical roles in antiviral immunity and disease severity in respiratory disease [105-107]. Along the same line, transcriptional upregulation of the protein kinase receptor (PKR, EIF2AK2), a cytoplasmatic pattern-recognition receptor could be observed. PKR is known to transduce RNA helicase (MDA5) dependent virus signals for type I IFN induction [108]. Interferon regulatory factor 7 (IRF7) is another key protein found strongly upregulated. Transcription factors IRF7 together with IRF3 regulate expression of early type I IFN and other proteins involved in the innate antiviral immune response (activation of IRF by cytosolic pattern recognition receptors) [109] (Supplementary Tables 1, 2, 3, 4). Signal transduction via PKR occurs mainly via NFKB and MAPK pathways (Role of PKR in Interferon induction and antiviral response) [110]. Another important intracellular patternrecognition receptor for viral RNA which was found to be upregulated by Echinaforce ${ }^{\circ}$ was the RNA helicase MDA5 (IFIH1) [78]. Furthermore, upregulation of the NF- $\mathrm{BB}$ subunits RelB and NFKB2/p52 was observed, which can promote downstream production of innate immunity chemokines (NF-KB activation by viruses, NF$\kappa B$ signaling) [111].

In line with our results showing activation of tonic IFN regulation of innate immunity gene responses, antiviral effects against influenza infection and activation of IFN pathways have also been demonstrated in vivo following Echinaforce ${ }^{\bullet}$ tincture treatment $[10,14,20]$. Along the same line, Echinaforce $e^{\bullet}$ treatment holds promise to reduce disease severity symptoms in SARSCoV2 patients by strengthening impaired IFN specific innate immune signaling $[64,70,71]$. Our in vitro results are also in line with observations in human studies ex vivo/in vivo showing increased immunomodulating as well as chemotactic neutrophil effects following Echinaforce $^{\circ}$ treatment $[10,22,23,112]$. For example, the antiviral ability of CXCL10 has been attributed to its chemoattractant effects which promote recruitment of natural killer cells [113-116] and neutrophils [113-116]. The latter illustrates that both neutrophils and inflammatory monocytes are intertwined in the immune system's anti-viral response [113-116]. Similar results were previously obtained in murine dendritic cells, illustrating that Echinaforce ${ }^{\bullet}$ stimulates cell mobility and chemotaxis and alters expression of cell adhesion and motility genes [117]. Other studies showed that Echinaforce may reverse the chemokine induction of virusinfected cells [11, 118-120]. Paradoxically, Echinaforce may induce cytokine and chemokine expression in uninfected cells, but suppress their expression upon virus infection or LPS stimulation [29, 118-120]. Similarly, Echinaforce ${ }^{\ominus}$ increased the transcription of TNF $\alpha$ in human monocytes, but reduced the LPS-stimulated TNF- $\alpha$ protein production [19]. Although studies suggest that this stimulatory effect may be the result of bacterialderived LPS and lipoproteins [26-29], our Echinaforce tincture contains no polysaccharides, neither endotoxins. Altogether, the latter suggests that its immunomodulatory effects are due to the active compounds present in the formulation [12, 19]. Similar activation of IFN innate immunity and viral protection has been observed in presence of avocado and apple extract [121, 122]. Interestingly, in the latter case, effects were attributed to oligomeric proanthocyanidins and lost with their monomeric form [122].

With respect to immunometabolism, mitochondrial metabolism shows a remarkable sensitivity to chemokine and IFN signaling [123, 124]. For example, ISG15 is an interferon-stimulated, ubiquitin-like protein which regulates mitochondrial homeostasis and targets various proteins involved in catabolic autophagy metabolism in the mitochondria (mitophagy) during infection [125, 126]. Moreover, mitochondrial changes in immunometabolism (glycolysis, the tricarboxylic acid (TCA) cycle, the pentose phosphate pathway, fatty acid oxidation, fatty acid synthesis and amino acid metabolism) strongly contribute in (re) shaping immunity and production of neutrophil extracellular traps (NETs) [127-130].

Next, phosphopeptide based kinome activity analysis revealed Echinaforce ${ }^{\bullet}$ specific activation of innate immunity and IFN signaling via multiple kinases, including JAK1, TEC, p38 MAPK (MAPK11, - 12, - 13, and - 14), JNK (MAPK8, - 9 and -10) and ERK1 kinases [131, 132]. JNK-STAT1 signaling induces various IFN responsive genes [133]. Moreover, JAK1 dependent regulation of downstream IFN and chemokine related gene expression after Echinaforce ${ }^{\bullet}$ treatment, could be reversed with the specific pharmacological JAK1 inhibitor filgotinib. TEC activation has important roles during innate immunity, i.e. IFN signaling via phosphorylation of JAK1 and JAK2 [131, 132], TLR signaling [134], assembly and activation of the caspase-8 inflammasome [135], macrophage survival [136], IL8 production [137], phagocytosis [138], and NFKB signaling [139]. p38 MAPK activation is involved in RIG-I dependent IFN signaling [140]. 
Various studies confirm involvement of these kinases in Echinacea biological action [19, 117, 141-144]. Alkylamides in the Echinaforce ${ }^{\circ}$ tincture were found to be responsible for MAPK effects upon binding to CB2 receptors leading to increased cAMP, P38/MAPK and JNK signaling, NFKB and ATF-2/CREB-1 activation [19]. Similarly, lipophilic extracts of Echinacea promoted murine dendritic cell maturation and mobility via the modulation of JNK, P38 MAPK and NFkB pathways [117, 141, 142]. Another study demonstrated a JAKSTAT1 dependent antiviral response of Tripterygium wilfordii (Thunder of God Vine) via the quinone methide triterpene celastrol [145].

Finally, genomewide epigenetic analysis of DNA methylation changes following Echinaforce ${ }^{\circ}$ treatment revealed almost 2000 DMP, enriched for immune disease and immunological pathways. Although the observed methylation changes are relatively small after $72 \mathrm{~h}$ treatment, cumulative effects can contribute in building an immune memory response by priming chromatin to mount faster and higher innate immune transcription upon re-stimulation of immune cells [103]. Besides the regulation of gene expression, DNA methylation is also involved in regulating alternative splicing, intron retention or promote cryptic transcription of non-annotated TSSs (TINATs) encoding immunogenic peptides which might prime an antiviral innate immune response [146149]. As such, it appears that Echinaforce ${ }^{\circ}$ treatment predominantly promotes epigenetic changes in innate immunity gene pathways and to a less extent of adaptive immunity responsive genes.

Besides, the higher global DNA hypermethylation observed after Echinaforce ${ }^{\bullet}$ treatment in LINE, SINE and LTR transposon repeats flanking endogenous retroviral sequences (HERVs), may be part evolutionary conserved (epi) genomic protective response against retrotransposition and viral infection [150, 151]. Similarly, IFN was shown to promote DNA methylation silencing of repeats and noncoding RNAs [39, 150, 152, 153]. Specific HERVs have been proposed to establish a protective effect against exogenous viral infections [154]. HERVs can act as IFN-inducible enhancers and have shaped the evolution of a transcriptional network underlying the IFN response [154-157]. Of particular interest, the MER41B family of ERV sequences contains a STAT1 binding site and regulates expression of IFN- $\gamma-$ responsive genes, such as absent in melanoma 2 (AIM2), and IFI6 [158, 159]. CRISPR-Cas9 deletion of a subset of these HERV elements in the human genome impaired expression of adjacent IFN-induced genes and revealed their involvement in the regulation of essential immune functions, including activation of the AIM2 inflammasome. Along the same line, DNA methylation inhibitors trigger an IFN response through viral mimicry via transcription of
dsRNAs of repetitive elements from HERVs which can activate RIG-I and MDA5 PRRs [150, 151]. RNA transcripts of HERVs can be reverse transcribed to generate ssDNA or expressed to generate proteins with viral signatures, much like the pathogen-associated molecular patterns of exogenous viruses, which allows them to be detected by the innate immune system $[160,161]$. In another example, silencing of the MLT1C49 HERV decreased expression of CXCL10 and CCL2 chemokines [162]. Finally, transcriptional changes of MLT1B and MER4D HERV transcription and innate immune signaling have also been described upon immunometabolic mitochondrial changes in protein kinase (PK)-M2 activity, which were counteracted by NFkB RelB [163]. From these examples, it appears that HERV regulatory sequences now constitute a dynamic reservoir of IFNinducible enhancers fueling genetic innovation in mammalian immune defenses [158, 164, 165].

Previous studies showed that Echinaforce ${ }^{\circ}$, besides its immunomodulating activities is also very active as a virucidal agent against viruses with membranes, i.e. HSV-1, respiratory syncytial virus, all tested human and avian strains of influenza A virus, as well as influenza B virus [166]. Along the same line, Echinacea polyphenol quercetin was found to inhibit the entry of HIV-luc/SARS pseudotyped virus into Vero E6 cells [167]. Similar protective effects could recently also be observed in a reconstituted nasal epithelium cell culture system by exposing Echinaforce -treated respiratory epithelium to droplets of HCoV-229E, SARS- or MERS-CoVs, imitating a natural infection [168]. In contrast Echinaforce ${ }^{\bullet}$ was found to be less effective against intracellular virus replication [168]. Consequently, virus already present within a cell could be refractory to the inhibitory effect of Echinaforce ${ }^{\circ}$, but virus particles shed into the extracellular fluids would be vulnerable. Therefore, the antiviral actions of the Echinaforce ${ }^{\ominus}$ may especially manifest during initial contact with the virus, i.e. at the inception of infection, and also during transmission of virus from infected cells.

\section{Conclusion}

In conclusion, our systems biology approach revealed that Echinaforce ${ }^{\circ}$ phytochemicals trigger multiple antiviral innate immunity pathways, involving tonic IFN signaling, activation of pattern recognition receptors, chemotaxis, immunometabolism and DNA hypermethylation of endogenous retroviral sequences. Further studies in preclinical respiratory infection models and double blind placebo-controlled intervention studies are needed to proof its prophylactic efficacy against common cold corona viruses $(\mathrm{CoV})$, Severe Acute Respiratory Syndrome (SARS)-CoV, and new occurring strains such as SARS-CoV-2, with strongly impaired interferon (IFN) type I response and weak innate antiviral defense. 


\section{Abbreviations}

IFN: Interferon; ISG: Interferon stimulated genes; PTK: Protein tyrosine kinase STK: Serine threonine kinase; JAK: Janus kinase; STAT: Signal transducer and activator of transcription factors; IRF : Interferon regulatory factor; $\mathrm{CXCL}$ : Chemokine (C-X-C motif) ligand (CXCL); HERV: Human endogenous retroviruses; DEG : Differentially expressed genes; CCL : CC chemokine receptor ligand; IL: Interleukin; TF: Transcription factor; PPI: Protein-protein interactions; DMP: Differentially methylated position; DMR: Differentially methylated region; LINE: Long interspersed nuclear DNA repeat elements; SINE: Short interspersed nuclear DNA repeat elements; LTR: Long terminal DNA repeat; $\mathrm{CGl}$ : $\mathrm{CpG}$ island

\section{Supplementary Information}

The online version contains supplementary material available at https://doi. org/10.1186/s12906-021-03310-5.

Additional file 1: Supplementary Table 1: Differentially expressed probes (FDR $<0.05$ and $\log F C>0.4$ ) after Echinaforce ${ }^{\circledast}$ tincture treatment. Additional file 2: Supplementary Table 2: Enriched Ingenuity canonical pathways of differentially expressed genes after Echinaforce ${ }^{\circledR}$ tincture treatment

Additional file 3: Supplementary Table 3: Ingenuity pathway enrichment analysis of diseases and biological functions of differentially expressed genes after Echinaforce ${ }^{\circledast}$ tincture treatment.

Additional file 4: Supplementary Table 4: STRING protein-proteininteraction plot and Metascape protein-protein-interaction MCODE network enrichment analysis of differentially expressed genes after Echinaforce ${ }^{\oplus}$ tincture treatment.

Additional file 5: Supplementary Table 5: PamGene upstream kinase analysis.

Additional file 6: Supplementary Table 6: Differentially methylated positions (FDR $<0.05$ and $\mid$ DeltaBetas $\mid>0.05$ ).

Additional file 7: Supplementary Table 7: Enriched Ingenuity canonical pathways of differentially methylated genes after Echinaforce ${ }^{\oplus}$ tincture treatment

\section{Acknowledgements}

We thank Bioforce group Dr. A. Vogel (Roggswil, Switzerland) for providing Echinaforce ${ }^{\oplus}$ reagents,

\section{Authors' contributions}

KD, WVB and AS conceived and designed the study. KD, CPN, LG performed the experiments and statistical data analysis. KD, CPN, LG AS and WVB interpreted the data. AS and GVC contributed reagents, EPIC array services and analysis tools. All authors wrote, evaluated revised and approved the final manuscript prior to submission.

\section{Funding}

KD, CPN and WVB are supported by FWO grants (G079614N, G059713N) and BOF NOI/DOCPRO/GOA grants (UA, FFP140094). The funding body had no role in the design of the study or collection, analysis, and interpretation of data and in writing the manuscript.

\section{Availability of data and materials}

Data are available on request. Transcriptome and DNA methylation data were uploaded to the Gene Expression Omnibus (GEO) database and have accession number: GSE117904.

\section{Declarations}

Ethics approval and consent to participate Not applicable.

\section{Consent for publication}

All authors approved the final manuscript for publication.

\section{Competing interests}

Author Andreas Suter was employed by the company A. Vogel Bioforce AG, Roggwil, Switzerland. The remaining authors declare that the research was conducted in the absence of any commercial or financial relationships that could be construed as a potential conflict of interest.

\section{Author details}

'Laboratory of Protein Chemistry, Proteomics and Epigenetic Signaling (PPES), Department of Biomedical Sciences, University of Antwerp (UA), Antwerp, Belgium. ${ }^{2}$ Center of Medical Genetics, Department of Biomedical Sciences, University of Antwerp (UA) and University Hospital Antwerp (UZA), Antwerp, Belgium. ${ }^{3}$ A. Vogel Bioforce AG, Roggwil, Switzerland.

Received: 9 May 2020 Accepted: 27 April 2021

Published online: 12 May 2021

\section{References}

1. Barnes J, et al. Echinacea species (Echinacea angustifolia (DC.) Hell., Echinacea pallida (Nutt.) Nutt.,Echinacea purpurea (L.) Moench): a review of their chemistry, pharmacology and clinical properties. J Pharm Pharmacol. 2005;57(8):929-54.

2. Barrett B. Medicinal properties of Echinacea: a critical review. Phytomedicine. 2003;10(1):66-86. https://doi.org/10.1078/09447110332164 8692.

3. Manayi A, Vazirian M, Saeidnia S. Echinacea purpurea: pharmacology, phytochemistry and analysis methods. Pharmacogn Rev. 2015;9(17):63-72.

4. Karsch-Volk M, Barrett B, Linde K. Echinacea for preventing and treating the common cold. JAMA. 2015;313(6):618-9.

5. Binns SE, Livesey JF, Arnason JT, Baum BR. Phytochemical variation in echinacea from roots and flowerheads of wild and cultivated populations. J Agric Food Chem. 2002;50(13):3673-87. https://doi.org/10.1021/jf011439t.

6. Hudson JB. Applications of the phytomedicine Echinacea purpurea (purple coneflower) in infectious diseases. J Biomed Biotechnol. 2012:769896.

7. Chicca A, et al. Synergistic immunomopharmacological effects of $\mathrm{N}$ alkylamides in Echinacea purpurea herbal extracts. Int Immunopharmacol. 2009;9(7-8):850-8

8. Dalby-Brown L, et al. Synergistic antioxidative effects of alkamides, caffeic acid derivatives, and polysaccharide fractions from Echinacea purpurea on in vitro oxidation of human low-density lipoproteins. J Agric Food Chem. 2005;53(24):9413-23.

9. Signer J, et al. In vitro virucidal activity of Echinaforce(R), an Echinacea purpurea preparation, against coronaviruses, including common cold coronavirus 229E and SARS-CoV-2. Virol J. 2020;17(1):136

10. Ritchie MR, et al. Effects of Echinaforce(R) treatment on ex vivo-stimulated blood cells. Phytomedicine. 2011;18(10):826-31.

11. Sharma M, et al. Induction of multiple pro-inflammatory cytokines by respiratory viruses and reversal by standardized Echinacea, a potent antiviral herbal extract. Antivir Res. 2009:83(2):165-70.

12. Sharma SM, et al. Bactericidal and anti-inflammatory properties of a standardized Echinacea extract (Echinaforce): dual actions against respiratory bacteria. Phytomedicine. 2010;17(8-9):563-8.

13. Sharma M, Schoop R, Hudson JB. Echinacea as an antiinflammatory agent: the influence of physiologically relevant parameters. Phytother Res. 2009; 23(6):863-7. https://doi.org/10.1002/ptr.2714.

14. Pleschka S, et al. Anti-viral properties and mode of action of standardized Echinacea purpurea extract against highly pathogenic avian influenza virus (H5N1, H7N7) and swine-origin H1N1 (S-OIV). Virol J. 2009;6:197.

15. Sharma M, Schoop R, Hudson JB. The efficacy of Echinacea in a 3-D tissue model of human airway epithelium. Phytother Res. 2010;24(6):900-4. https:// doi.org/10.1002/ptr.3051.

16. Canlas J, Hudson JB, Sharma M, Nandan D. Echinacea and trypanasomatid parasite interactions: growth-inhibitory and anti-inflammatory effects of Echinacea. Pharm Biol. 2010;48(9):1047-52. https://doi.org/10.3109/138802 00903483468

17. Woelkart K, et al. Bioavailability and pharmacokinetics of Echinacea purpurea preparations and their interaction with the immune system. Int J Clin Pharmacol Ther. 2006:44(9):401-8.

18. Sharma M, et al. The potential use of Echinacea in acne: control of Propionibacterium acnes growth and inflammation. Phytother Res. 2011; 25(4):517-21. 
19. Gertsch J, Schoop R, Kuenzle U, Suter A. Echinacea alkylamides modulate TNF-alpha gene expression via cannabinoid receptor CB2 and multiple signal transduction pathways. FEBS Lett. 2004;577(3):563-9. https://doi.org/1 0.1016/j.febslet.2004.10.064

20. Vimalanathan $S$, et al. Prevention of influenza virus induced bacterial superinfection by standardized Echinacea purpurea, via regulation of surface receptor expression in human bronchial epithelial cells. Virus Res. 2017;233: 51-9.

21. Vimalanathan S, Schoop R, Hudson J. High-potency anti-influenza therapy by a combination of Echinacea purpurea fresh herb and root tinctures. J Appl Pharmaceut Sci. 2013;3:1-5

22. Brinkeborn RM, Shah DV, Degenring FH. Echinaforce and other Echinacea fresh plant preparations in the treatment of the common cold. A randomized, placebo controlled, double-blind clinical trial. Phytomedicine. 1999;6(1):1-6.

23. Jawad M, et al. Safety and efficacy profile of Echinacea purpurea to prevent common cold episodes: A randomized, double-blind, Pacebo-Controlled Trial. Evid Based Complement Alternat Med. 2012:841315.

24. Raduner S, Majewska A, Chen JZ, Xie XQ, Hamon J, Faller B, et al. Alkylamides from Echinacea are a new class of cannabinomimetics. Cannabinoid type 2 receptor-dependent and -independent immunomodulatory effects. J Biol Chem. 2006;281(20):14192-206. https:// doi.org/10.1074/jbc.M601074200.

25. Bruni R, Brighenti V, Caesar LK, Bertelli D, Cech NB, Pellati F. Analytical methods for the study of bioactive compounds from medicinally used Echinacea species. J Pharm Biomed Anal. 2018;160:443-77. https://doi.org/1 0.1016/j.jpba.2018.07.044

26. Pugh ND, Jackson CR, Pasco DS. Total bacterial load within Echinacea purpurea, determined using a new PCR-based quantification method, is correlated with LPS levels and in vitro macrophage activity. Planta Med. 2013;79(1):9-14.

27. Pugh ND, et al. The majority of in vitro macrophage activation exhibited by extracts of some immune enhancing botanicals is due to bacterial lipoproteins and lipopolysaccharides. Int Immunopharmacol. 2008;8(7):102332.

28. Tamta $\mathrm{H}$, et al. Variability in in vitro macrophage activation by commercially diverse bulk echinacea plant material is predominantly due to bacterial lipoproteins and lipopolysaccharides. J Agric Food Chem. 2008;56(22): 10552-6.

29. Todd DA, et al. Ethanolic Echinacea purpurea extracts contain a mixture of cytokine-suppressive and cytokine-inducing compounds, including some that originate from Endophytic Bacteria. PLoS One. 2015;10(5):e0124276.

30. Dunning MJ, et al. Beadarray: R classes and methods for Illumina beadbased data. Bioinformatics. 2007;23(16):2183-4.

31. Barbosa-Morais NL, et al. A re-annotation pipeline for Illumina BeadArrays: improving the interpretation of gene expression data. Nucleic Acids Res 2010;38(3):e17.

32. Ritchie ME, et al. Limma powers differential expression analyses for RNAsequencing and microarray studies. Nucleic Acids Res. 2015;43(7):e47.

33. Reiner A, Yekutieli D, Benjamini Y. Identifying differentially expressed genes using false discovery rate controlling procedures. Bioinformatics. 2003;19(3): 368-75.

34. Dunning M, L.A.a.E.M., illuminaHumanv4.db: Illumina HumanHT12v4 annotation data (chip illuminaHumanv4). 2015, R package version 1.26.0.

35. Kramer A, et al. Causal analysis approaches in ingenuity pathway analysis. Bioinformatics. 2014;30(4):523-30. https://doi.org/10.1093/bioinformatics/ btt703.

36. Zhou Y, et al. Metascape provides a biologist-oriented resource for the analysis of systems-level datasets. Nat Commun. 2019;10:1-10.

37. Han H, Cho JW, Lee S, Yun A, Kim H, Bae D, et al. TRRUST v2: an expanded reference database of human and mouse transcriptional regulatory interactions. Nucleic Acids Res. 2018;46(D1):D380-6. https://doi.org/10.1093/ nar/gkx1013.

38. Bader GD, Hogue CW. An automated method for finding molecular complexes in large protein interaction networks. BMC Bioinform. 2003;4:2.

39. Roulois D, Loo Yau H, Singhania R, Wang Y, Danesh A, Shen SY, et al. DNADemethylating agents target colorectal Cancer cells by inducing viral mimicry by endogenous transcripts. Cell. 2015;162(5):961-73. https://doi. org/10.1016/j.cell.2015.07.056.

40. Safaei J, et al. Prediction of 492 human protein kinase substrate specificities. Proteome Sci. 2011;9(Suppl 1):S6.
41. Chirumamilla CS, et al. Profiling activity of cellular kinases in migrating Tcells. Methods Mol Biol. 2019;1930:99-113. https://doi.org/10.1007/978-1-493 9-9036-8_13.

42. Szarc Vel Szic K, et al. Epigenetic silencing of triple negative breast cancer hallmarks by Withaferin A. Oncotarget. 2017;8(25):40434-53. https://doi. org/10.18632/oncotarget.17107.

43. Assenov Y, Müller F, Lutsik P, Walter J, Lengauer T, Bock C. Comprehensive analysis of DNA methylation data with RnBeads. Nat Methods. 2014;11(11): 1138-40. https://doi.org/10.1038/nmeth.3115.

44. Teschendorff $A E$, et al. A beta-mixture quantile normalization method for correcting probe design bias in Illumina Infinium 450 k DNA methylation data. Bioinformatics. 2013;29(2):189-96.

45. Hansen KD, IlluminaHumanMethylationEPICmanifest: Manifest for Illumina's EPIC methylation arrays. 2016, R package version 0.3.0.

46. Milenkovic $D$, et al. Dietary flavanols modulate the transcription of genes associated with cardiovascular pathology without changes in their DNA methylation state. PLoS One. 2014;9(4):e95527.

47. Chirumamilla CS, Palagani A, Kamaraj B, Declerck K, Verbeek MWC, Oksana $\mathrm{R}$, et al. Selective glucocorticoid receptor properties of GSK866 analogs with cysteine reactive warheads. Front Immunol. 2017;8:1324. https://doi.org/1 0.3389/fimmu.2017.01324.

48. Milenkovic D, et al. A systems biology network analysis of nutri (epi) genomic changes in endothelial cells exposed to epicatechin metabolites. Sci Rep. 2018;8(1):15487.

49. Michalska A, et al. A positive feedback amplifier circuit that regulates interferon (IFN)-stimulated gene expression and controls type I and type II IFN responses. Front Immunol. 2018;9:1135.

50. Mogensen TH. IRF and STAT transcription factors - from basic biology to roles in infection, protective immunity, and primary Immunodeficiencies. Front Immunol. 2018:9:3047.

51. Verhelst J, et al. Interferon-inducible protein $M \times 1$ inhibits influenza virus by interfering with functional viral ribonucleoprotein complex assembly. J Virol. 2012;86(24):13445-55.

52. Verhelst J, Hulpiau P, Saelens X. Mx proteins: antiviral gatekeepers that restrain the uninvited. Microbiol Mol Biol Rev. 2013;77(4):551-66.

53. Smith SE, et al. Interferon-induced transmembrane protein 1 restricts replication of viruses that enter cells via the plasma membrane. J Virol. 2019;93(6):e0200318(1-13).

54. Bailey CC, et al. IFITM-family proteins: the Cell's first line of antiviral defense. Annu Rev Virol. 2014;1:261-83.

55. Szklarczyk D, et al. STRING v11: protein-protein association networks with increased coverage, supporting functional discovery in genome-wide experimental datasets. Nucleic Acids Res. 2019;47(D1):D607-13.

56. Zhou Y, et al. Metascape provides a biologist-oriented resource for the analysis of systems-level datasets. Nat Commun. 2019;10(1):1523.

57. Hussein HAM, Akula SM. miRNA-36 inhibits KSHV, EBV, HSV-2 infection of cells via stifling expression of interferon induced transmembrane protein 1 (IFITM1). Sci Rep. 2017;7(1):17972.

58. Brogaard L, Heegaard PMH, Larsen LE, Mortensen S, Schlegel M, Dürrwald R, et al. Late regulation of immune genes and microRNAs in circulating leukocytes in a pig model of influenza A (H1N2) infection. Sci Rep. 2016;6(1): 21812. https://doi.org/10.1038/srep21812.

59. Gough DJ, et al. Constitutive type I interferon modulates homeostatic balance through tonic signaling. Immunity. 2012;36(2):166-74.

60. Divangahi $M$, et al. Trained immunity, tolerance, priming and differentiation: distinct immunological processes. Nat Immunol. 2021;22(1):2-6.

61. Ivashkiv LB. IFNgamma: signalling, epigenetics and roles in immunity, metabolism, disease and cancer immunotherapy. Nat Rev Immunol. 2018; 18(9):545-58. https://doi.org/10.1038/s41577-018-0029-z.

62. Wilk $\mathrm{AJ}$, et al. A single-cell atlas of the peripheral immune response in patients with severe COVID-19. Nat Med. 2020;26(7):1070-6.

63. Zhang JY, Wang XM, Xing X, Xu Z, Zhang C, Song JW, et al. Single-cell landscape of immunological responses in patients with COVID-19. Nat Immunol. 2020;21(9):1107-18. https://doi.org/10.1038/s41590-020 0762-x.

64. Blanco-Melo D, et al. Imbalanced host response to SARS-CoV-2 drives development of COVID-19. Cell. 2020;181(5):1036-45 e9.

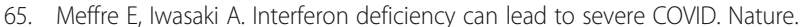
2020;587(7834):374-6.

66. Stertz $\mathrm{S}$, Hale BG. Interferon system deficiencies exacerbating severe pandemic virus infections. Trends Microbiol. 2021. 
67. Bastard P, et al. Autoantibodies against type I IFNs in patients with lifethreatening COVID-19. Science. 2020;370(6515):eabd4585.

68. Zhang Q, et al. Inborn errors of type I IFN immunity in patients with lifethreatening COVID-19. Science. 2020;370(6515):eabd4585.

69. Lopez L, et al. Dysregulated Interferon Response Underlying Severe COVID19. Viruses. 2020:12.

70. Hadjadj J, Yatim N, Barnabei L, Corneau A, Boussier J, Smith N, et al. Impaired type I interferon activity and inflammatory responses in severe COVID-19 patients. Science. 2020;369(6504):718-24. https://doi.org/10.1126/ science.abc6027

71. Sa Ribero M, et al. Interplay between SARS-CoV-2 and the type I interferon response. PLoS Pathog. 2020;16(7):e1008737.

72. van Dam PA, et al. SARS-CoV-2 and cancer: are they really partners in crime? Cancer Treat Rev. 2020;89:102068.

73. Paolucci S, et al. EBV DNA increase in COVID-19 patients with impaired lymphocyte subpopulation count. Int J Infect Dis. 2020;104:315-9.

74. Arsenault R, Griebel P, Napper S. Peptide arrays for kinome analysis: new opportunities and remaining challenges. Proteomics. 2011;11(24):4595-609.

75. Dussaq A, et al. Mechanistic parameterization of the Kinomic signal in peptide arrays. J Proteomics Bioinform. 2016;9(5):151-7.

76. Labots $\mathrm{M}$, et al. Evaluation of a tyrosine kinase peptide microarray for tyrosine kinase inhibitor therapy selection in cancer. Exp Mol Med. 2016; 48(12):e279.

77. Baharani A, Trost B, Kusalik A, Napper S. Technological advances for interrogating the human kinome. Biochem Soc Trans. 2017:45(1):65-77. https://doi.org/10.1042/BST20160163.

78. Mesev EV, LeDesma RA, Ploss A. Decoding type I and III interferon signalling during viral infection. Nat Microbiol. 2019;4(6):914-24. https://doi.org/10.103 8/s41564-019-0421-x.

79. Kar UK, Joosten LAB. Training the trainable cells of the immune system and beyond. Nat Immunol. 2020;21(2):115-9.

80. Novakovic B, et al. Beta-Glucan reverses the epigenetic state of LPS-induced immunological tolerance. Cell. 2016;167(5):1354-68 e14.

81. Saeed S, et al. Epigenetic programming of monocyte-to-macrophage differentiation and trained innate immunity. Science. 2014;345(6204): 1251086.

82. Szarc Vel Szic K, et al. From inflammaging to healthy aging by dietary lifestyle choices: is epigenetics the key to personalized nutrition? Clin Epigenet. 2015;7:33.

83. Vanden Berghe W, et al. Keeping up NF-kappaB appearances: epigenetic control of immunity or inflammation-triggered epigenetics. Biochem Pharmacol. 2006;72(9):1114-31. https://doi.org/10.1016/j.bcp.2006.07.012.

84. Vanden Berghe W. Epigenetic impact of dietary polyphenols in cancer chemoprevention: lifelong remodeling of our epigenomes. Pharmacol Res. 2012;65(6):565-76. https://doi.org/10.1016/j.phrs.2012.03.007.

85. Milagro Fl, et al. Dietary factors, epigenetic modifications and obesity outcomes: progresses and perspectives. Mol Asp Med. 2013;34(4):782-812.

86. Remely $\mathrm{M}$, et al. Therapeutic perspectives of epigenetically active nutrients Br J Pharmacol. 2015;172(11):2756-68.

87. Koch $A$, et al. Analysis of DNA methylation in cancer: location revisited. Nat Rev Clin Oncol. 2018;15(7):459-66.

88. Milenkovic D, et al. (-)-Epicatechin metabolites promote vascular health through epigenetic reprogramming of endothelial-immune cell signaling and reversing systemic low-grade inflammation. Biochem Pharmacol. 2019: 113699.

89. Dayeh T, Volkov P, Salö S, Hall E, Nilsson E, Olsson AH, et al. Genome-wide DNA methylation analysis of human pancreatic islets from type 2 diabetic and non-diabetic donors identifies candidate genes that influence insulin secretion. PLoS Genet. 2014;10(3):e1004160. https://doi.org/10.1371/journal. pgen. 1004160

90. Langie SAS, Moisse M, Szarc vel Szic K, van der Plas E, Koppen G, de Prins S, et al. GLI2 promoter hypermethylation in saliva of children with a respiratory allergy. Clin Epigenetics. 2018;10(1):50. https://doi.org/10.1186/ s13148-018-0484-1.

91. Deaton AM, et al. Cell type-specific DNA methylation at intragenic CpG islands in the immune system. Genome Res. 2011;21(7):1074-86.

92. Pulloor NK, et al. Human genome-wide RNAi screen identifies an essential role for inositol pyrophosphates in type-I interferon response. PLoS Pathog. 2014;10(2):e1003981.

93. Kutateladze TG. Histone deacetylation: IP4 is an epigenetic coregulator. Nat Chem Biol. 2012;8(3):230-1.
94. Burton A, Azevedo C, Andreassi C, Riccio A, Saiardi A. Inositol pyrophosphates regulate JMJD2C-dependent histone demethylation. Proc Natl Acad Sci U S A. 2013;110(47):18970-5. https://doi.org/10.1073/pnas.13 09699110.

95. Meng $\mathrm{H}$, et al. DNA methylation, its mediators and genome integrity. Int J Biol Sci. 2015;11(5):604-17.

96. Papin C, et al. Combinatorial DNA methylation codes at repetitive elements. Genome Res. 2017;27(6):934-46.

97. Nanan KK, Ocheltree C, Sturgill D, Mandler MD, Prigge M, Varma G, et al. Independence between pre-mRNA splicing and DNA methylation in an isogenic minigene resource. Nucleic Acids Res. 2017;45(22):12780-97. https://doi.org/10.1093/nar/gkx900.

98. Jones PA. Functions of DNA methylation: islands, start sites, gene bodies and beyond. Nat Rev Genet. 2012;13(7):484-92. https://doi.org/10.1038/ nrg3230.

99. Kim D, Shivakumar M, Han S, Sinclair MS, Lee YJ, Zheng Y, et al. Populationdependent intron retention and DNA methylation in breast Cancer. Mol Cancer Res. 2018;16(3):461-9. https://doi.org/10.1158/1541-7786.MCR-17-0227.

100. Mancini RJ, et al. Directing the immune system with chemical compounds. ACS Chem Biol. 2014;9(5):1075-85.

101. Muralidharan S, Mandrekar P. Cellular stress response and innate immune signaling: integrating pathways in host defense and inflammation. J Leukoc Biol. 2013;94(6):1167-84. https://doi.org/10.1189/jlb.0313153.

102. Piasecka A, Jedrzejczak-Rey N, Bednarek P. Secondary metabolites in plant innate immunity: conserved function of divergent chemicals. New Phytol. 2015;206(3):948-64.

103. Kamada R, et al. Interferon stimulation creates chromatin marks and establishes transcriptional memory. Proc Natl Acad Sci U S A. 2018;115(39): E9162-71.

104. Howitz KT, Sinclair DA. Xenohormesis: sensing the chemical cues of other species. Cell. 2008;133(3):387-91.

105. Tang BM, et al. A novel immune biomarker IFI27 discriminates between influenza and bacteria in patients with suspected respiratory infection. Eur Respir J. 2017:49(6):1602098(1-12).

106. Zhao X, et al. IFITM genes, variants, and their roles in the control and pathogenesis of viral infections. Front Microbiol. 2018;9:3228.

107. Gao J, Zhu X, Wu M, Jiang L, Wang F, He S. IFI27 may predict and evaluate the severity of respiratory syncytial virus infection in preterm infants. Hereditas. 2021;158(1):3. https://doi.org/10.1186/s41065-020-00167-5.

108. Pham AM, et al. PKR transduces MDA5-dependent signals for type I IFN induction. PLoS Pathog. 2016;12(3):e1005489.

109. Andrilenas KK, et al. DNA-binding landscape of IRF3, IRF5 and IRF7 dimers: implications for dimer-specific gene regulation. Nucleic Acids Res. 2018; 46(5):2509-20.

110. Takada Y, Ichikawa H, Pataer A, Swisher S, Aggarwal BB. Genetic deletion of PKR abrogates TNF-induced activation of IkappaBalpha kinase, JNK, Akt and cell proliferation but potentiates p44/p42 MAPK and p38 MAPK activation. Oncogene. 2007;26(8):1201-12. https://doi.org/10.1038/sj.onc.1209906.

111. Sun SC. The non-canonical NF-kappaB pathway in immunity and inflammation. Nat Rev Immunol. 2017;17(9):545-58.

112. Roesler J, et al. Application of purified polysaccharides from cell cultures of the plant Echinacea purpurea to test subjects mediates activation of the phagocyte system. Int J Immunopharmacol. 1991;13(7):931-41.

113. Sokol CL, Luster AD. The chemokine system in innate immunity. Cold Spring Harb Perspect Biol. 2015;7(5):a016303(1-19).

114. Yuan J, et al. CXCL10 inhibits viral replication through recruitment of natural killer cells in coxsackievirus B3-induced myocarditis. Circ Res. 2009;104(5):628-38.

115. Freeman BE, et al. Cytokine-mediated activation of NK cells during viral infection. J Virol. 2015;89(15):7922-31.

116. Lodoen MB, Lanier LL. Natural killer cells as an initial defense against pathogens. Curr Opin Immunol. 2006;18(4):391-8. https://doi.org/10.1016/j. coi.2006.05.002.

117. Yin SY, et al. Stimulatory effect of Echinacea purpurea extract on the trafficking activity of mouse dendritic cells: revealed by genomic and proteomic analyses. BMC Genomics. 2010;11:612.

118. Sharma M, Arnason JT, Hudson JB. Echinacea extracts modulate the production of multiple transcription factors in uninfected cells and rhinovirus-infected cells. Phytother Res. 2006;20(12):1074-9.

119. Cech NB, et al. Echinacea and its alkylamides: effects on the influenza Ainduced secretion of cytokines, chemokines, and PGE (2) from RAW 264.7 macrophage-like cells. Int Immunopharmacol. 2010;10(10):1268-78. 
120. Sharma M, Arnason JT, Burt A, Hudson JB. Echinacea extracts modulate the pattern of chemokine and cytokine secretion in rhinovirus-infected and uninfected epithelial cells. Phytother Res. 2006;20(2):147-52. https://doi. org/10.1002/ptr.1824.

121. Wu YH, et al. Avocado (Persea americana) fruit extract (2R,4R)-1,2,4trihydroxyheptadec-16-yne inhibits dengue virus replication via upregulation of NF-kappaB-dependent induction of antiviral interferon responses. Sci Rep. 2019;9(1):423.

122. Snyder DT, et al. Oral delivery of oligomeric procyanidins in apple poly(R) enhances type I IFN responses in vivo. J Leukoc Biol. 2014;95(5):841-7.

123. Raniga K, Liang C. Interferons: reprogramming the metabolic network against viral infection. Viruses. 2018;10(1):36(1-21).

124. Monlun M, Hyernard C, Blanco P, Lartigue L, Faustin B. Mitochondria as molecular platforms integrating multiple innate immune Signalings. J Mol Biol. 2017;429(1):1-13. https://doi.org/10.1016/j.jmb.2016.10.028.

125. Zhang Y, et al. The in vivo ISGylome links ISG15 to metabolic pathways and autophagy upon listeria monocytogenes infection. Nat Commun. 2019;10(1):5383.

126. Albert M, et al. ISG15, a small molecule with huge implications: regulation of mitochondrial homeostasis. Viruses. 2018;10(11):629(1-18).

127. O'Neill LA, Kishton RJ, Rathmell J. A guide to immunometabolism for immunologists. Nat Rev Immunol. 2016;16(9):553-65.

128. Viola A, et al. The metabolic signature of macrophage responses. Front Immunol. 2019;10:1462.

129. Kumar S, Dikshit M. Metabolic insight of neutrophils in health and disease. Front Immunol. 2019;10:2099.

130. Azevedo EP, Rochael NC, Guimarães-Costa AB, de Souza-Vieira TS, Ganilho J, Saraiva EM, et al. A metabolic shift toward pentose phosphate pathway is necessary for amyloid fibril- and Phorbol 12-Myristate 13-acetate-induced neutrophil extracellular trap (NET) formation. J Biol Chem. 2015;290(36): 22174-83. https://doi.org/10.1074/jbc.M115.640094.

131. Takahashi-Tezuka $M$, et al. Tec tyrosine kinase links the cytokine receptors to PI-3 kinase probably through JAK. Oncogene. 1997;14(19):2273-82.

132. Yamashita $Y$, et al. Tec and Jak2 kinases cooperate to mediate cytokinedriven activation of c-fos transcription. Blood. 1998;91(5):1496-507.

133. Lee, S. and S. Vasudevan, JNK MAPK Regulates IFN-Stimulated Genes and Cell Adhesion in Chemoresistant, Quiescent Leukemic Cells. BioRxiv, 2019. doi.org/10.1101/689570.

134. Tampella G, Kerns HM, Niu D, Singh S, Khim S, Bosch KA, et al. The Tec kinase-regulated Phosphoproteome reveals a mechanism for the regulation of inhibitory signals in murine macrophages. J Immunol. 2015;195(1):246-56. https://doi.org/10.4049/jimmunol.1403238.

135. Zwolanek F, et al. The non-receptor tyrosine kinase Tec controls assembly and activity of the noncanonical caspase-8 inflammasome. PLoS Pathog. 2014;10(12):e1004525.

136. Melcher M, Unger B, Schmidt U, Rajantie IA, Alitalo K, Ellmeier W. Essential roles for the Tec family kinases Tec and Btk in M-CSF receptor signaling pathways that regulate macrophage survival. J Immunol. 2008;180(12):804856. https://doi.org/10.4049/jimmunol.180.12.8048.

137. Wang GQ, Yang XY, Jia YT, Xia ZF. Tec kinase mediating IL-8 transcription in monocytes stimulated with LPS. Inflammation. 2009;32(4):265-9. https://doi. org/10.1007/s10753-009-9129-z.

138. Jongstra-Bilen J, et al. Dual functions of Bruton's tyrosine kinase and Tec kinase during Fcgamma receptor-induced signaling and phagocytosis. J Immunol. 2008;181(1):288-98.

139. Wang F, Zhang W, Wang C, Fang X, Cheng H, Liu S, et al. Inhibitor of Tec kinase, LFM-A13, decreases pro-inflammatory mediators production in LPSstimulated RAW264.7 macrophages via NF-kappaB pathway. Oncotarget. 2017;8(21):34099-110. https://doi.org/10.18632/oncotarget.16212.

140. Mikkelsen SS, et al. RIG-I-mediated activation of p38 MAPK is essential for viral induction of interferon and activation of dendritic cells: dependence on TRAF2 and TAK1. J Biol Chem. 2009;284(16):10774-82.

141. Wang CY, et al. Genomics and proteomics of immune modulatory effects of a butanol fraction of echinacea purpurea in human dendritic cells. BMC Genomics. 2008:9:479.

142. Li Y, et al. Echinacea pupurea extracts promote murine dendritic cell maturation by activation of JNK, p38 MAPK and NF-kappaB pathways. Dev Comp Immunol. 2017;73:21-6.

143. Sullivan AM, et al. Echinacea-induced macrophage activation. Immunopharmacol Immunotoxicol. 2008;30(3):553-74.

144. Fu A, et al. Echinacea purpurea extract polarizes M1 macrophages in murine bone marrow-derived macrophages through the activation of JNK. J Cell Biochem. 2017;118(9):2664-71.
145. Yu JS, et al. Celastrol inhibits dengue virus replication via up-regulating type I interferon and downstream interferon-stimulated responses. Antivir Res. 2017;137:49-57.

146. Brocks D, et al. DNMT and HDAC inhibitors induce cryptic transcription start sites encoded in long terminal repeats. Nat Genet. 2017;49(7):1052-60.

147. Shayevitch $R$, et al. The importance of DNA methylation of exons on alternative splicing. RNA. 2018;24(10):1351-62.

148. Greenberg MVC, Bourc'his D. The diverse roles of DNA methylation in mammalian development and disease. Nat Rev Mol Cell Biol. 2019;20(10): 590-607.

149. Savan R. Alternative splicing in innate antiviral immunity. J Interf Cytokine Res. 2018;38(8):317-8. https://doi.org/10.1089/jir.2018.29010.rsa.

150. Chiappinelli KB, et al. Inhibiting DNA methylation causes an interferon response in Cancer via dsRNA including endogenous retroviruses. Cell. 2015:162(5):974-86.

151. Yu Q, Carbone CJ, Katlinskaya Y, Zheng H, Zheng K, Luo M, et al. Type I interferon controls propagation of long interspersed element-1. J Biol Chem. 2015;290(16):10191-9. https://doi.org/10.1074/jbc.M114.612374.

152. Leonova Kl, et al. p53 cooperates with DNA methylation and a suicidal interferon response to maintain epigenetic silencing of repeats and noncoding RNAs. Proc Natl Acad Sci U S A. 2013;110(1):E89-98.

153. Licht JD. DNA methylation inhibitors in Cancer therapy: the immunity dimension. Cell. 2015;162(5):938-9.

154. Grandi N, Tramontano E. Human endogenous retroviruses are ancient acquired elements still shaping innate immune responses. Front Immunol. 2018:9:2039.

155. Buzdin AA, Prassolov V, Garazha AV. Friends-enemies: endogenous retroviruses are major transcriptional regulators of human DNA. Front Chem. 2017:5:35.

156. Sun $X$, et al. Transcription factor profiling reveals molecular choreography and key regulators of human retrotransposon expression. Proc Natl Acad Sc U S A. 2018;115(24):E5526-35.

157. Johnson WE. Origins and evolutionary consequences of ancient endogenous retroviruses. Nat Rev Microbiol. 2019;17(6):355-70.

158. Tokuyama M, Kong Y, Song E, Jayewickreme T, Kang I, Iwasaki A. ERVmap analysis reveals genome-wide transcription of human endogenous retroviruses. Proc Natl Acad Sci U S A. 2018;115(50):12565-72. https://doi. org/10.1073/pnas.1814589115.

159. Ito J, et al. Systematic identification and characterization of regulatory elements derived from human endogenous retroviruses. PLoS Genet. 2017; 13(7):e1006883.

160. Hurst TP, Magiorkinis G. Activation of the innate immune response by endogenous retroviruses. J Gen Virol. 2015;96(Pt 6):1207-18.

161. Briard B, Place DE, Kanneganti TD. DNA sensing in the innate immune response. Physiology (Bethesda). 2020;35(2):112-24.

162. Canadas I, et al. Tumor innate immunity primed by specific interferonstimulated endogenous retroviruses. Nat Med. 2018;24(8):1143-50. https:// doi.org/10.1038/s41591-018-0116-5.

163. Stone OA, el-Brolosy M, Wilhelm K, Liu X, Romão AM, Grillo E, et al. Loss of pyruvate kinase $M 2$ limits growth and triggers innate immune signaling in endothelial cells. Nat Commun. 2018;9(1):4077. https://doi.org/10.1038/s414 67-018-06406-8.

164. Chuong EB, Elde NC, Feschotte C. Regulatory evolution of innate immunity through co-option of endogenous retroviruses. Science. 2016;351(6277): 1083-7.

165. Meyer TJ, et al. Endogenous retroviruses: with us and against us. Front Chem. 2017:5:23.

166. Hudson J, Vimalanathan S. Echinacea-A source of potent antivirals for respiratory virus infections. Pharmaceuticals. 2011:4(7):1019-31.

167. Yi L, Li Z, Yuan K, Qu X, Chen J, Wang G, et al. Small molecules blocking the entry of severe acute respiratory syndrome coronavirus into host cells. J Virol. 2004;78(20):11334-9. https://doi.org/10.1128/JVI.78.20.11334-11339.2004.

168. Signer, J., et al., In vitro antiviral activity of Echinaforce ${ }^{\circledR}$, an Echinacea purpurea preparation, against common cold coronavirus 229E and highly pathogenic MERS-CoV and SARS-CoV. BMC Virology journal, 2020: p. DOl: https://doi.org/10.21203/rs.2.24724/v2.

\section{Publisher's Note}

Springer Nature remains neutral with regard to jurisdictional claims in published maps and institutional affiliations. 